\title{
Le rôle des virtuoses dans la valorisation de la musique soviétique Examen de la presse communiste française
}

\author{
Thomas THISSELIN
}

La valorisation en France entre 1945 et 1956 de la musique soviétique s'opère à travers les événements ou les articles de presse consacrés aux virtuoses soviétiques. La musique est valorisée par des arts ou des pratiques connexes, néanmoins parties intégrantes de la musique : l'enseignement soviétique, la danse, le cinéma... La période stalinienne d'après-guerre élargie à l'immédiat après Staline représente l'acmé de la dictature stalinienne et reste encore aujourd'hui la plus mystérieuse de l'histoire soviétique. Cette période correspond par ailleurs, en URSS, à un moment d'exacerbation de la politique culturelle dite du « réalisme socialiste » dans le domaine des arts, et spécialement de la musique. Cela exigeait de l'artiste une représentation véridique, historiquement concrète de la réalité dans son développement révolutionnaire, son devoir étant de participer à la lutte idéologique et à l'éducation des masses dans l'esprit du socialisme.

Limiter la question de la réception de la musique soviétique aux réactions et aux discussions que cette musique suscitait dans les revues savantes serait ne pas prendre en compte la manière dont elle a interrogé, en tant que modèle, la sphère de la consommation musicale française en général. Il s'agit ici de ne pas interpréter la réception de la musique soviétique en France à travers celle d'un compositeur ou d'une œuvre. Les virtuoses sont ainsi valorisés comme des intermédiaires.

La force d'un tel travail réside en la masse d'informations recueillies et organisées à partir de toutes les publications nationales assimilées communistes de l'époque, chambres d'écho de ce qui se passait alors en URSS et riches en informations sur les carrières et les représentations des virtuoses. Bien qu'elles s'adressent à des 
SLOVO

250 Les Voyages lointains des écrivains polonais $\left(\mathrm{XX}^{\mathrm{e}}-\mathrm{XXI}^{\mathrm{e}}\right.$ siècles $)-\mathrm{n}^{\circ} 51$

lectorats différents et n'aient pas les mêmes périodicités ou degrés d'expertise, ces publications sympathisantes nous informent sur la place de la musique soviétique dans cette promotion (elles militent pour les échanges culturels, considérant que le resserrement des liens culturels entre les peuples crée les conditions les plus favorables pour leur entente mutuelle, une lutte commune pour la paix).

Le rôle joué par les performances des virtuoses soviétiques en France est facile à identifier. Plus qu'une question de génie individuel, sans négliger cet aspect, les revues communistes mettent en avant la maitrise et le talent de tous ces artistes dont l'origine commune serait l'école à laquelle ils ont été formés et, par-delà, le régime qui a créé les conditions de cette floraison ${ }^{1}$. Leurs succès indéniables nous renseignent sur une efficacité des méthodes et de l'organisation de l'enseignement mais aussi sans doute sur des conditions de vie artistique dans lesquelles ils pouvaient s'épanouir ; méthodes qui donnent aux élèves, peut-on lire, une technique telle, qu' ils expriment les musiques les plus difficiles sans effort.

\section{L'école du violon russe}

Après la révolution d'Octobre, un enseignement du violon se refonde. Le centre de gravité de l'école russe, jusqu'alors à Saint-Pétersbourg, se déplace vers Moscou. Après avoir fui la révolution, Nathan Milstein, passé par la classe de Leopold Auer, vécut la terreur et la privation à Odessa - également un pôle d'attraction à l'époque. Il se retrouva à Paris en 1920 avec son ami d'infortune le pianiste Vladimir Horowitz. Il y rencontre Eugène Ysaÿe, grand maître de l'école franco-belge aux côtés de Henri Vieuxtemps notamment. Ce dernier enseignait à Moscou et à Saint-Pétersbourg, et s'était produit au Théâtre du Palais Ioussoupov entre autres. Cette empreinte française en Russie, participant à l'essor de l'école russe, n'est pas sans rappeler la production en France de toute une série de russes (peintres et producteurs de théâtre et de cinéma par exemple). Certains d'entre eux comme Chagall, Soutine ou Diaghilev vécurent la plus grande partie de leur vie en France et devinrent même les représentants de l'école française.

Le violon a contribué en tant qu'objet spectaculaire, à la popularisation de la musique soviétique. Milstein sera considéré comme «l'invincible dont la perfection de son jeu est telle qu'il est difficile de formuler de légères critiques sans paraître "le censeur grognon" ${ }^{2}$ ». Jascha Heifetz quant à lui comme le « dieu de

1.La Nouvelle Critique, «"Le Concert des Maîtres de l'art” », Cahiers du communisme, « Les échanges culturels avec le monde socialiste ».

2. Ce Soir, « Milstein l'invincible ». 
la musique », sans geste inutile 3 . On reviendra notamment sur les concerts qu'il donna pendant la guerre pour les soldats et les blessés, les suivant sur la plupart des terrains d'opération.

La suprématie soviétique dans l'exécution musicale serait due au changement de régime et à la jeunesse d'artistes qui ont foi en un régime favorable aux arts. Déjà en 1937, David Ö̈strakh faisait la promotion du régime ${ }^{4}$. On retrouve de nombreux éloges à l'égard de Oïstrakh, qui atteindrait la grandeur de la simplicité par le naturel de son jeu ; une forme de jeunesse peut-on lire ${ }^{5}$. La virtuosité n'aurait en aucun cas contaminé sa musicalité, la technique restant au service d'une musique humaniste, fidèle au texte. Il est fait mention de l'intérêt d'Oistrakh pour la poésie et notamment son utilité pour les premières classes des conservatoires de musique, une telle lecture étant révélatrice chez l'élève de son sens du rythme et de la forme. Il parle de sa vie quotidienne à un correspondant de L'Humanité, partagée entre pédagogie et travail personnel, n'attachant pas moins d'importance à l'enrichissement culturel. Selon lui, la façon de travailler et le contact avec le public prévalent.

Ö̈strakh devient un ambassadeur de sa patrie et le porte-parole édifiant des musiciens du pays du socialisme. Il finit second au concours Wieniawski, derrière Ginette Neveu, avant de remporter le premier prix en 1937 au concours Ysaÿe de Bruxelles. Il acquiert une notoriété internationale et l'URSS décide alors d'envoyer en bloc des ambassadeurs; Kogan, Richter, Guilels et Oisstrakh, malgré lui, puisqu'il n'avait aucune accointance avec le régime. Le titre honorifique d'artiste du peuple lui est pourtant décerné et L'Humanité multipliera le report de critiques élogieuses extraites volontairement de journaux de tous bords. Remarqué

3. Les Lettres françaises, « Rentrée civile d'un dieu de la musique : Jascha Heifetz ».

4. La Pravda, Izvestia, « Le triomphe des musiciens soviétiques ».

5.Les Lettres françaises, «David Oïstrakh est à Paris », «En écoutant David Oïstrakh », L'Humanité, «Pour "L'Humanité" David Oïstrakh évoque sa vie quotidienne », «Triomphal succès de David Ö̈strakh à Chaillot», «Le triomphal concert. David Ö̈strakh au Palais de Chaillot », « La presse unanime rend hommage au talent de David Ö̈strakh », Les Lettres françaises, « Le miracle David Oïstrakh », L'Humanité, «David Oïstrakh "artiste du peuple” », «Prestiges et vertus du virtuose », Les Lettres françaises, «La Sonate à Kreutzer de Beethoven », L'Humanité, «Suivez le guide... de l'Académie Charles-Cros », « David Ö̈strakh : "Le rideau de fer est américain” », FranceU.R.S.S., « Le Concerto de Brahms par David Oïstrakh », L'Humanité, « Yehudi Menuhin à Moscou... David Ö̈strakh à New-York », «À la salle Pleyel dimanche David Oïstrakh s'est confirmé le plus grand violoniste vivant », Les Lettres françaises, « Ö̈strakh à Paris ». 
SLOVO

252 Les Voyages lointains des écrivains polonais $\left(\mathrm{XX}^{\mathrm{e}}-\mathrm{XXI}^{\mathrm{e}}\right.$ siècles $)-\mathrm{n}^{\circ} 51$

pour les mêmes raisons, son fils Igor perpétuera cette école ${ }^{6}$. Les journaux d'octobre 1954 annonceront les premiers récitals de musiciens soviétiques dont ceux d'Igor Ö̈strakh? .

David Ö̈strakh était avec Dmitri Kabalevski et Lev Oborine à Paris en 1953 à l'occasion d'une conférence de presse dans les salons de l'hôtel George $\mathrm{V}$ avec l'Agence littéraire parisienne ${ }^{8}$. Kabalevski évoque sa jeunesse, l'école musicale, le conservatoire, alors qu'il étudie le piano, il suit en même temps les cours d'une école de dessin et d'un institut économique. Il souligne aussi la généralisation dans les écoles d'enseignements dispensés par des étudiants. Pour les œuvres qu'ils composent, les musiciens font part de leurs projets au ministère de la Culture, qui leur passe des commandes. Si l'œuvre est bonne, elle est aussitôt éditée et diffusée et son auteur reçoit de nouveaux honoraires. Des prix Staline ont même été décernés à des élèves du conservatoire. Ö̈strakh, évoquant les kolkhozes, soutenait combien tout le peuple soviétique se passionnait pour la musique symphonique et les conférences d'éducation musicale. Ö̈strakh et Oborine soulignaient combien les études musicales étaient beaucoup plus longues en URSS qu'en France, renforçant ainsi l'importance accordée à la culture générale.

La circulation des virtuoses a été facilitée par la nature individuelle de la performance. Kabalevski rappelait que depuis le premier concours Chopin en 1927, les musiciens soviétiques avaient participé à 17 concours internationaux de musique, et que 63 prix leur avaient été décernés, dont 16 premiers prix. Lev Oborine, alors considéré comme « un des trois plus grands pianistes de l'Union soviétique » aux interprétations fidèles se produisit à Paris avec le violoniste Igor Bezrodni dont la maîtrise technique et la sonorité étaient remarquées ${ }^{9}$. Il avait remporté le

6.L'Humanité, «Igor Oisstrakh remporte le concours de violon de Poznan. Blanche Tarjus 3' », L'Humanité-dimanche, «Le violoniste Igor Oïstrakh et la presse britannique », Les Lettres françaises, «Tel Père, tel fils... Igor Oïstrakh », « Brève rencontre... », L'Humanité, « Le triomphe d'Igor Oïstrakh à la salle Pleyel », Les Lettres françaises, «Voici pourquoi Igor Oistrakh joue si bien... », Cabiers du communisme, « Les échanges culturels avec le monde socialiste ».

7. Chroniques étrangères. U.R.S.S., « La saison théâtrale et musicale de Moscou ».

8. L'Humanité-dimanche, «Trois prestigieux ambassadeurs de l'art soviétique à Paris », L'Humanité, « Avant les deux concerts de Chaillot Kabalevski, Ö̈strakh et Oborine au C.N.E. », Les Lettres françaises, « Rencontre des musiciens », « Regrets et rétrospectives », Cabiers du communisme, « Les échanges culturels avec le monde socialiste ».

9.L'Humanité, «Dimanche soir à Pleyel Léon Oborine Igor Bezrodni merveilleux musiciens », Ce Soir, « Pour la première fois depuis de nombreuses années, deux grands virtuoses soviétiques, L. Oborine (pianiste) et I. Bezrodni (violoniste) sont à Paris où 
concours international des jeunes à Prague en 1949 et le concours Bach de Leipzig en 1950. Oborine se félicitait quant à lui de jouer partout en Union soviétique, pour des ouvriers, des intellectuels, des kolkhoziens, des étudiants, des soldats... Il sera par ailleurs enchanté de jouer aussi bien à Pleyel, à Chaillot, à la maison de la Pensée que devant des auditoires de travailleurs à Ivry et chez Renault, ou pour des enfants du XVIII ${ }^{e}$ arrondissement. L'accueil de la province n'aurait pas été moins chaleureux, que ce soit à Marseille, Grenoble ou Montpellier. Il a reçu le prix Staline, après avoir remporté en 1927 le concours international Chopin. À propos des méthodes d'enseignement appliquées en Union soviétique, Oborine évoque le soin qu'apportent les professeurs à inculquer aux élèves, non seulement une technique éprouvée, mais surtout une discipline artistique et le respect absolu de l'œuvre interprétée. Il revient également sur ce qui constituerait l'essentiel à savoir que n'importe quel fils d'ouvrier ou de kolkhozien aux aptitudes remarquées avait la possibilité de devenir un grand artiste.

Hélène Jourdan-Morhange voyait les violonistes soviétiques comme vainqueurs de tous les concours auxquels ils allaient participer ${ }^{10}$. À l'occasion du concours Wieniawski de 1952 remporté par Igor Ö̈strakh et dont les 3 premiers prix avaient été remportés par des soviétiques (Julian Sitowiecki second, Marina Jaszwili et Olga Parchomienko troisièmes), le professeur Orvid, directeur du conservatoire de Moscou s'exprima ${ }^{11}$. Il revint sur la prise en charge des enfants par les pensions d'État dans les hameaux et les villages aussi bien que dans les villes afin que le nombre d'initiés soit accru. Il ne serait ainsi pas rare de découvrir un paysan ou un ouvrier devenir un futur virtuose. Dans les premières classes, on leur apprend le solfège en même temps que l'alphabet. Ils rejoignent ensuite des écoles décennales

ils joueront dimanche à Pleyel », « À Pleyel : Oborine et Bezrodni deux (très) grands virtuoses soviétiques », «Léo Oborine et Igor Bezrodni au Palais de Chaillot », FranceU.R.S.S., «L. Oborine et I. Bezrodni emportent du public français un chaleureux souvenir », L'Humanité, « Le 29 juin à Chaillot avec David Ö̈strakh Lev Oborine un très grand pianiste », « Nouveau triomphal succès hier soir à Chaillot. David Oïstrakh et Lev Oborine », « Après leur triomphe à Chaillot David Ö̈strakh et Lev Oborine joueront ce soir à Lille. Dernier concert à Pleyel le 3 juillet », « David Ö̈strakh et Lev Oborine à l'Opéra de Lille », «Les adieux de Lev Oborine et de David Ö̈strakh », Les Lettres françaises, « La Sonate à Kreutzer de Beethoven ».

10. Les Lettres françaises, « Tel Père, tel fils... Igor Oïstrakh », « Brève rencontre... », « Voici pourquoi Igor Ö̈strakh joue si bien... ».

11. Ce Soir, «La violoniste Blanche Tarjus obtient le troisième prix international Wieniawski », Les Lettres françaises, « Concours international de violon Wieniawski », «Palmarès Wieniawski ». 
SLOVO

254 Les Voyages lointains des écrivains polonais $\left(\mathrm{XX}^{\mathrm{e}}-\mathrm{XXI}^{\mathrm{e}}\right.$ siècles $)-\mathrm{n}^{\circ} 51$

où la musique et l'instruction vont de pair. Ils apprennent le violon avec de grands élèves déjà récompensés, dont la méthode serait donc excellente. Il est par exemple fait allusion aux méthodes d'archet qui se transmettent fidèlement depuis Leopold Auer, gage de traditions entretenues. Jusqu'à l'âge de 15 ou 16 ans malgré tout, l'élève peut choisir son métier, les études supérieures et les études musicales étant enseignées en parallèle. Si l'élève a opté pour la carrière musicale, il entre au conservatoire après une sévère sélection où, là encore, les études générales étaient couplées à un enseignement plus développé de l'instrument et de l'harmonie. Les études sont longues mais ne rebuteraient ni les parents ni les élèves, puisque les leçons étaient gratuites. L'État accordait des pensions et des bourses, pour les plus doués, afin de supprimer tout souci matériel. Les virtuoses et même certains élèves, avaient par ailleurs, grâce à la sollicitude de l'État, la possibilité d'utiliser des chefs d'œuvres de la lutherie du monde entier, et notamment de la lutherie russe, rassemblés dans la collection d'instruments d'État créée dès 1925 dont le fonds initial fut la collection de Stradivarius du tsar Alexandre $\mathrm{I}^{\text {er }}$ et celle de Pavel Tretiakov.

\section{Les virtuoses comme témoins vivants de la culture soviétique}

En faisant parler des personnalités telles que Oborine, Bezrodni ou Yampolski, les journalistes transformaient les virtuoses en témoins non seulement de l'art soviétique mais surtout de la culture soviétique ${ }^{12}$. Yampolski insiste aussi sur le fait que l'Union soviétique ne fait pas d'enfants prodiges mais plutôt des hommes capables de devenir aussi bien de grands solistes que des ingénieurs, à l'image de Bezrodni qui pouvait aussi bien rentrer à l'Institut d'aéronautique. Igor Oistrakh déclarait quant à lui ne pas savoir à 15 ans s'il allait être ingénieur ou violoniste. En 1941, quand commencèrent les bombardements de Moscou, les usines furent transportées loin du front. Dans les trains qui emportaient le matériel de première importance se trouvaient les jeunes élèves de l'École de musique de Moscou, dont Bezrodni, qui avait alors onze ans. Les études pouvaient ainsi se poursuivre loin du front.

La qualité du jeu des artistes soviétiques, leur modestie face au texte et l'absence totale de recherche de l'effet, « en opposition aux excès de beaucoup d'interprètes occidentaux, plus soucieux de conquérir un public à leur jeu personnel que de servir

12. Les Lettres françaises, « L. Oborine, W. Jampolski et I. Bezrodni ou la musique soviétique à Paris », L'Humanité, «Le jeu de haute qualité des virtuoses soviétiques », Les Lettres françaises, « Le C.N.E. reçoit les deux artistes soviétiques : L. Oborine et I. Bezrodni ». 
la musique $\gg$ qualifient le jeu des virtuoses soviétiques ${ }^{13}$. On trouve ainsi dénoncé l'antisoviétisme qui voulait montrer chez l'Union soviétique un «impérialisme culturel », au même titre que la concurrence capitaliste - assimilée à une mise en scène hollywoodienne - qui conforterait l'individualisme d'interprètes devenus des marchandises commerciales. L'éducation du public soviétique serait plus élevée dans son ensemble, impliquant des exigences plus hautes dans les interprétations.

Quant au violoncelliste Mstislav Rostropovitch ${ }^{14}$, alors accueilli en France par Maurice Maréchal, aux chanteurs Zaria Doloukhanova ${ }^{15}$, Ivan Kozlovski, Yelena Kruglikova, Andrey Ivanov, Mark Reyzen ${ }^{16}$ et Ivan Petrov ${ }^{17}$, au chef d'orchestre Jascha Horenstein ${ }^{18}$, à la pianiste Nina Yemelyanova ${ }^{19}$, et même au marionnettiste Serge Obrastzov ${ }^{20}$ également à l'honneur à l'occasion de sa venue à la Comédie Caumartin, ils ne feraient que confirmer les qualités techniques des soviétiques. À propos d'Ivan Petrov, ce dernier était arrivé à un degré de vérité historique et de perfection. Ses interprétations de Boris de Moussorgski ou de

13. La Nouvelle Critique, «Le "Chant des forêts" de Chostakovitch et la musique soviétique ».

14. L'Humanité, «La rencontre de deux grands violoncellistes », « Avant son récital de vendredi prochain salle Gaveau, le grand violoncelliste soviétique Rostropovitch était samedi au Conservatoire », «Rostropovitch et ses pairs », « Rostropovitch a tenu une gageure à la salle Gaveau », Les Lettres françaises, « Rostropovitch ».

15. Les Lettres françaises, « Pourquoi Doloukhanova chante si bien... ».

16. L’Humanité, « Découverte d'“Eugène Onéguine” » opéra favori des Soviétiques... et inconnu des Français ».

17. L'Humanité, «Le chanteur soviétique Ivan Petrov est arrivé à Paris », «Ivan Petrov à l'Opéra », «Ce soir à l'Opéra. Ivan Petrov deux fois Prix Staline à 34 ans sera Boris Godounov », « Ivan Petrov triomphe à l'Opéra dans “Boris Godounov" », «Ivan Petrov inoubliable Boris Godounov», «Quelques opinions de la critique sur Ivan Petrov », « Hier, réception à l'Opéra en l'honneur d'Ivan Petrov », Les Lettres françaises, «Ivan Pétrov : Un Boris sans pareil », L'Humanité-dimanche, «Ivan Petrov à l'Opéra », L'Humanité, « Ivan Petrov incomparable Méphisto à l'Opéra », Les Lettres françaises, «Ivan Petrov. Un grand Méphisto qui conduit le bal », L'Humanité, « Immense succès d'Ivan Petrov au Palais de Chaillot », «Ivan Petrov artiste soviétique », FranceU.R.S.S., «Ivan Petrov dans Boris Godounov à l'Opéra », Les Lettres françaises, « Retour d'Ivan Petrov ».

18. Ce Soir, « Jascha Horenstein à Paris ».

19. L'Humanité, « Nina Emilianova jouera ce soir au Conservatoire de Paris ».

20.Les Lettres françaises, «Voici pourquoi Obrastzov vous fait rire... », L'Humanité, «Les marionnettistes français reçoivent Serge Obrastzov», «Les marionnettes de Serge Obrastzov et celles d'Yves Joly se sont rencontrées à la maison de la Chimie ». 
SLOVO

256 Les Voyages lointains des écrivains polonais $\left(\mathrm{XX}^{\mathrm{e}}-\mathrm{XXI}^{\mathrm{e}}\right.$ siècles $)-\mathrm{n}^{\circ} 51$

Méphisto de Gounod sont très remarquées. Son jeu serait empreint d'une entière compréhension du satanisme conforme à la tradition lyrique instituée par le génie mélodique et théâtral de Gounod. L'interprète se place systématiquement sur un plan essentiellement humain et ne sacrifie jamais à quelque beauté formelle. Il s'agit du résultat d'une assimilation profonde, qualifiée de scientifique, de chaque œuvre interprétée. L'art soviétique est bâti sur la confiance en l'homme, son travail, son intelligence et sa compréhension.

Si nous sommes ici concentrés sur la nature individuelle de la performance, notons malgré tout que la Chorale Piatnitski comptait parmi les grandes écoles soviétiques de chant choral. Au même titre que le Chœur russe de Voronèje dirigé par Massalitinov ou le Chœur académique Svechnikov, elle est à l'opposé de l'école classique et traditionnaliste caractérisée par ce dernier, fidèle interprète du folklore. La Chorale Piatnitski marquait l'apogée d'un modernisme dans le chant choral soviétique, avec une façon de chanter qui n'était pas très académique à proprement parler, l'émission du son étant gutturale, non sans rappeler le chant choral paysan.

\section{Les concours comme dispositif de médiatisation}

Le concours Eugène Ysaÿe est un moyen pour les virtuoses soviétiques de contribuer activement à la promotion de leur musique. Leonid Kogan en constitua l'illustration. Il était valorisé par les journalistes communistes comme l'incarnation de la méthode soviétique, célébré pour sa rigueur, sa sûreté technique, son naturel et son honnêteté artistique qui auraient élevé tout ce qu'il touche sans jamais sacrifier à un effet spectaculaire ${ }^{21}$. Il remporte le concours de 1951 et Mikhail Vayman obtient

21.L'Humanité, «Un violoniste soviétique remporte le "Grand Prix musical de la Reine Elisabeth de Belgique" », Ce Soir, «Le disque que vous pouvez offrir pour les étrennes: Capriccio espagnol de Rimski-Korsakov, Fantaisie sur des thèmes de "Carmen" », L'Humanité, «Le grand violoniste soviétique Léonide Kogan sera pour la première fois à Paris le 14 février à Chaillot», «En transit à Paris l'année dernière le célèbre violoniste soviétique Léonide Kogan jouera lundi soir à Chaillot », « Un grand violoniste Léonide Kogan jouera ce soir à Chaillot », « Hier soir au Palais de Chaillot un très grand violoniste Léonide Kogan s'est révélé aux Parisiens », «Léonide Kogan à Toulouse », Les Lettres françaises, «Léonid Kogan à Chaillot », «Léonid Kogan ou la maîtrise de l'archet », «Léonid Kogan aux Champs-Élysées », L'Humanité, «Accord parfait de la critique et du public : Léonide Kogan est un très grand violoniste », « Léonide Kogan prépare son deuxième récital », «Léonide Kogan reçu par la Commission musicale de France-U.R.S.S. », «Léonide Kogan à Nancy », Les Lettres françaises, «Léonid Kogan ou la maîtrise de l'archet », L'Humanité, « Le violoniste soviétique Léonide Kogan vient à Paris révéler un concerto inédit de Vivaldi », « Le violoniste soviétique Léonide Kogan 
le deuxième prix, une compétition où les russes s'adjurent 4 des 11 prix. Kogan fera plusieurs remarques à la suite du concours, critiquant le fait que les marchandises vendues à Bruxelles proviennent des États-Unis, le manque de fréquentation des spectacles dans la capitale, leurs coûts très chers et leur programmation peu intéressante ${ }^{22}$. Il déclare ne pas avoir pu voir un seul film belge, les écrans étant « envahis par les films américains » dont le sujet est dûment reproduit par une réclame qui n'est rien d'autre qu'un véritable « musée d'instruction criminelle » selon l'artiste. Il se fait l'écho, de façon volontaire ou contre son gré, du discours de propagande soviétique. Adopter le mode de vie américain freinerait donc l'essor d'une production nationale, portant atteinte à son originalité et détournant la population de son héritage. Dénonçant de manière caricaturale « "la nourriture spirituelle" qu'on fait avaler à la population belge », Kogan condamnait, conformément à l'idéologie du régime soviétique, la décadence capitaliste d'une civilisation naturaliste.

Pour le violoniste Jacques Thibaud, le niveau de la prestation de Kogan était « inimaginable ». Ce dernier indique que la presse ne tarda pas à reconnaître l'école de violon soviétique comme la meilleure du monde. Les interprètes sont salués comme des représentants de cette culture qui se veut progressiste, Kogan rappelant la mission qui anime tous les représentants de l'Union, à savoir représenter leur culture musicale et y faire honneur. Kogan aurait été étonné par certaines réactions belges. Les étudiants du conservatoire de Bruxelles par exemple, se questionnaient sur la vie musicale en URSS. Ses interlocuteurs auraient envié un système qui assumait la plus grande partie des frais d'études et qui mettait à disposition des instruments précieux. L'ignorance manifestée sur la vie en Union soviétique a été l'occasion d'attiser davantage la haine envers les pays capitalistes et la presse bourgeoise. Les artistes soviétiques partent avec le sentiment que les belges veulent s'ouvrir au système soviétique, chose pourtant rendue impossible avec le rideau de fer.

L'article envoyé par Jo Heifetz, américaine cultivée et fille de l'illustre violoniste aux Lettres françaises se fera l'écho du concours Ysaÿe et de cette participation sensationnelle des virtuoses soviétiques ${ }^{23}$. Elle s'attarde sur les deuxième (Mikhail Vayman), cinquième (Alexeï Gorokhov) et septième (Olga Kaverzneva)

ou le triomphe de la technique et du goût », Les Lettres françaises, « Un grandissime violoniste », « Léonid Kogan aux Champs-Élysées ».

22. Ce Soir, «Des virtuoses du monde entier se sont retrouvés à Bruxelles », Sovietskoë Isskoustvo.

23. Les Lettres françaises, « Un Grand Prix international de violon sous le signe de la paix ». 
SLOVO

258 Les Voyages lointains des écrivains polonais $\left(\mathrm{XX}^{\mathrm{e}}-\mathrm{XXI}^{\mathrm{e}}\right.$ siècles $)-\mathrm{n}^{\circ} 51$

places. David Ö̈strakh, premier prix au concours de 1937, était membre du jury. Il viendra à Paris en 1953 (afin de siéger au jury du concours Marguerite-LongJacques-Thibaud) et la présence cette même année de Kogan (à l'occasion de concerts à Chaillot) poussèrent les spectateurs à la comparaison ${ }^{24}$. Depuis Ö̈strakh, jamais un violoniste de cette classe n'avait été entendu.

La suprématie soviétique dans le domaine de l'interprétation serait donc due à une absence de formalisme ou de surenchère esthétique dont souffriraient les musiciens élevés dans une ambiance capitaliste. On trouve mention du succès des artistes au festival du mai Florentin entre autres ${ }^{25}$. La prestation acclamée d'Émile Gilels est évoquée et de nombreux articles reviendront sur cette personnalité, prix Staline en 1946 et vainqueur du concours Ysaÿe en $1938^{26}$. La presse italienne remarquait l'attitude consciencieuse avec laquelle les soviétiques se préparaient tout en visitant la ville, traduisant un sentiment de profonde responsabilité à l'égard du peuple soviétique mais aussi une curiosité à découvrir la culture propre d'un pays. Né d'une famille d'employés, Gilels se serait révélé comme un artiste d'une grande maturité qui dès l'adolescence avait privilégié sa culture musicale et sa culture générale, étudiant au conservatoire la philosophie, l'histoire

24. France-U.R.S.S., « Le violoniste soviétique Léonide Kogan en France ».

\section{Sovietskoë Isskoustvo, Izvestia.}

26. L'Humanité, «Un des plus grands pianistes soviétiques vient à Paris dans quelques jours », « Le grand pianiste soviétique Émile Guillels qui sera dans quelques jours à Paris remporta son premier triomphe à 15 ans », « Le grand pianiste soviétique Émile Guillels est arrivé hier à Paris », « Avant son concert de demain salle Pleyel Émile Guillels évoque pour vous quelques souvenirs parisiens », « Hier soir, salle Pleyel Grand succès du récital Émile Guillels », Les Lettres françaises, «Émile Guillels à Paris », L'Humanité, «Émile Guillels, Michèle Boussinot, Aimé Doniat participeront dimanche à Pleyel à l'hommage à Stalingrad », «Un très grand artiste: Émile Guillels fait l'unanimité de la presse parisienne », «Émile Guillels donnera un récital d'œuvres soviétiques modernes jusqu'à présent jamais exécutées en France », « Immense succès du récital d'Émile Guillels à la salle Pleyel », «Émile Guillels (magicien du piano) a connu à Pleyel un nouveau triomphe », France-U.R.S.S., « Un grand pianiste soviétique à Paris », Les Lettres françaises, «Émile Guillels ou l'égalité de la perfection», «Guillels au C.N.E. », L'Humanité, «Dernier concert d'Émile Guillels mardi à Chaillot», « Brillante réception en l'honneur des artistes soviétiques », L'Humanité-dimanche, «Claude Roy a écouté pour vous Émile Guillels », L'Humanité, «Le grand pianiste soviétique Émile Guillels viendra le mois prochain à Besançon », « Le pianiste soviétique Émile Guillels est à nouveau l'hôte de Paris », Chroniques étrangères. U.R.S.S., « La saison théâtrale et musicale de Moscou », L'Humanité, «Émile Guillels et André Cluytens », «Au Palais de Chaillot le 14 juin l'illustre pianiste soviétique Émile Guillels donnera un unique concert », « Émile Guillels triomphe à Chaillot ». 
et les sciences. De là, le directeur du théâtre Pariso Votto aurait déclaré en sortant du concert n'avoir encore jamais vu d'artistes atteindre une telle maturité, prenant avec un tel sentiment de responsabilité leurs récitals. À nouveau des critiques de tous horizons sont privilégiées par notre presse communiste.

La pianiste Marguerite Long et le violoniste Jacques Thibaud fondaient en 1943 un concours qui allait devenir international. Pour la première fois en 1953, 10 ans après donc, des artistes de l'Union soviétique y participaient. Neuf musiciens avaient demandé à l'ambassade de France à Moscou un visa de séjour pour participer au concours à Paris: Oborine et Ö̈strakh (membres de leurs jurys respectifs), Tchoulvki, Fedorova, Malinin, Yampolski, Nehauss, Chkolnikova et Sobolevski ${ }^{27}$. Le compositeur Kabalevski dirigera la délégation soviétique. Il sera évoqué à de nombreuses reprises le premier grand prix alors jugé indiscutable et le prix Ginette Neveu décernés à Nelly Chkolnikova dont le jeu avait été comparé à celui d'Heifetz ${ }^{28}$. Elle s'est empressée de dire qu'elle n'était qu'étudiante et non une vedette, beaucoup étant comme elle à Moscou, un propos similaire est d'ailleurs trouvé au sujet du violoniste Gérard Jarry qui gagna le concours à 15 ans, qualifié plutôt de petit provincial, très doué et très travailleur ${ }^{29}$. Elle avait alors devant elle encore quatre années d'études (une année de conservatoire et trois années d'Aspiranture). On revient sur sa scolarité « normale » en parallèle à un apprentissage complet de la musique lui permettant de comprendre son art en interprète sensible et en artiste cultivée. Il est aussi fait mention d'un Guarnerius qui lui avait été confié par le gouvernement pour le concours.

27. L'Humanité, « Neuf musiciens soviétiques bientôt à Paris ? », « David Oïstrakh et Lev Oborine à Paris ».

28. Les Lettres françaises, «Un concours brillant et juste », L'Humanité-dimanche, «Une heure avec la violoniste soviétique Nelly Chkolnikova. Premier grand prix du concours international Jacques Thibaud », France-U.R.S.S., « Grâce au concours Jacques Thibaud Marguerite Long, une violoniste de 24 ans a ébloui Paris qui pourtant ne se laisse pas séduire facilement », «Les violonistes soviétiques l'ont prouvé : la musique passionne "le grand public" », L'Humanité, « Nelly Chkolnikova qui conquit Paris cet été triomphe à Moscou », « Nelly Chkolnikova et Evgeny Malinine joueront demain soir au théâtre des Champs-Élysées », «Un émouvant et magnifique concert : celui de Nelly Chkolnikova et Evgeny Malinine au théâtre des Champs-Élysées », Les Lettres françaises, « Hommage à J. Thibaud par les jeunes lauréats soviétiques », L'Humanité, «En attendant le concert de Nelly Chkolnikova », « Dernier récital de Nelly Chkolnikova aux Champs-Élysées », Les Lettres françaises, « La sereine tranquillité de Nelly Chkolnikova », France-U.R.S.S., «Violon et piano ».

29. Ce Soir dimanche, «15 ans. Gérard Jarry (Prix Marguerite Long Jacques Thibaud) sera dans quelques années, grâce à son travail l'un des grands noms de la musique ». 
SLOVO

Les Voyages lointains des écrivains polonais $\left(\mathrm{XX}^{\mathrm{e}}-\mathrm{XXI}^{\mathrm{e}}\right.$ siècles $)-\mathrm{n}^{\circ} 51$

Le concours constitue un dispositif de médiatisation non seulement pour ses organisateurs mais pour les virtuoses soviétiques eux-mêmes. Kabalevski revient sur le fait qu'à aucun moment il ne pourrait être trouvé dans une œuvre soviétique un soupçon d'agressivité à l'égard d'autres peuples. Quelques années auparavant Irina Politkovskaïa, également au conservatoire de Moscou, rapportait déjà les discussions entre étudiants de son cours, centrées sur l'amitié et le refus de prôner l'attaque des peuples voisins ${ }^{30}$. Chkolnikova, alors âgée de 24 ans lors de sa participation au concours Marguerite-Long-Jacques-Thibaud, reviendra sur la préparation, expliquant que les violonistes soviétiques avaient au préalable été présélectionnés en URSS ${ }^{31}$. Sur les trente candidats, Raphaël Sobolevski et elle avaient remporté le droit d'aller représenter l'Union au concours. Sobolevski, dont la grande impression a été relevée lors de la première journée, obtiendra un second prix.

Kabalevski explique qu'en URSS il existe 659 écoles musicales dont 19 dans les villes principales. L'Humanité justifie les succès des virtuoses soviétiques par les conditions de la vie musicale en URSS ${ }^{32}$. Les écoles musicales elles-mêmes s'appuient sur un réseau immense de cercles d'amateurs. Il n'est pas de palais de la culture, de kolkhoze, d'usine ou de grande entreprise qui ne possède son cercle d'amateurs : une véritable école de musique, dotée du matériel nécessaire où sont donnés par des artistes et pédagogues éminents, des conférences éducatives et des concerts spécialisés. Il est regretté qu'en France certains enfants aient pratiquement à renoncer à la musique parce que les cours gratuits sont rares, insuffisants et les instruments très onéreux.

Quant au concours du même nom pour piano, la très haute tenue internationale del'événement est remarquée ${ }^{33}$. Cependant les résultats avaient provoqué des remous

30. France-U.R.S.S., « Pour la paix, le peuple soviétique répond : présent ! ».

31.L'Humanité, « La $1^{\text {re }}$ journée du Concours International Marguerite Long Jacques Thibaud. Le jeune violoniste soviétique R. Sobolevski a fait une grande impression sur le public », «2 $2^{\mathrm{e}}$ Journée de concours Salle Gaveau », « Le Concours Long - Thibaud. Finale disputée aujourd'hui par 8 sélectionnés dont Sobolevsky et N. Chkolnikova », «Triomphal succès des musiciens soviétique au concours Long-Thibaud», «Les commentaires de la presse sur le triomphal succès de Nelly Chkolnikova », France-U.R.S.S., «Quinze jours durant danseurs, chanteurs et musiciens soviétiques ont enchanté la France $\gg$.

32. L'Humanité, «Quelques raisons des succès des jeunes musiciens soviétiques ».

33. L'Humanité, «Les pianistes s'affrontent à leur tour salle Gaveau », « 40 pianistes restent en ligne au concours Marguerite-Long », Les Lettres françaises, « Moins brillant et moins juste ». 
dans l'assistance ${ }^{34}$. Jacques Thibaud, n'assimilait pas ce concours à une compétition musicale et regrettait que pour des raisons politiques, les deux grands prix (violon et piano) n'aient pas été décernés à deux soviétiques. Evgueni Malinin et le français Philippe Entremont reçurent deux seconds prix. Là encore la presse communiste ne manque pas de citer les critiques unanimes de la presse, quelle que soit l'appartenance politique ${ }^{35}$. On relève également les déplacements des musiciens en France: Chkolnikova et Malinin ont donné un concert de musique russe avec l'Orchestre Philharmonique de Bordeaux sous la direction de Jean Fournet, quant à Ö̈strakh, Oborine et Yampolski ils se sont vus remettre à Lille une médaille d'or frappée aux armes de la ville. Les virtuoses polonais - Barbara Hesse-Bukowska et Lydia Gritchtol (piano), Edward Statkiewicz (violon) - sont également à l'honneur à Bruay-en-Artois (chez les mineurs), à la maison des Syndicats de Bruay, à la maison du Peuple de Lens, ou à Marseille ${ }^{36}$. Lydia Gritchtol précisant qu'elle était originaire d'une famille de mineurs, on observe avec une rhétorique similaire combien les musiciens polonais auraient également, grâce à la musique, démontré avec éclat tout ce que le régime de leur pays offrait comme possibilités aux jeunes talents.

La disparition de Jacques Thibaud en 1953 est évoquée ${ }^{37}$. Il est fait mention de son art fait de simplicité et de ses leçons amicales, s'attachant à ses élèves et les encourageant avec optimisme. Il défendait l'écriture nationale française. Décrit comme fidèle aux partitions qu'il interprétait, il n'en forçait jamais le sens et respectait le message du compositeur. Les plus grands musiciens soviétiques signèrent un texte hommage où il est mentionné son vif intérêt pour la Russie. Les représentations de Jacques Thibaud en Union soviétique (la dernière fois en 1936) remportaient un invariable succès. Ses concerts avec des exécutants soviétiques, à

34. L'Humanité, « Au concours Marguerite Long. Remous dans le public après la décision du jury », « Finale mouvementée au concours Marguerite Long - Jacques Thibaud ».

35.L'Humanité, «Dimanche, à Pleyel $2^{\mathrm{e}}$ concert avec les lauréats du concours Long-Thibaud $\gg$.

36. L'Humanité, « Les jeunes virtuoses soviétiques à Bordeaux », L'Humanité-dimanche, «Musiciens en voyage... Maîtres et disciples soviétiques à travers la France », «De Bruay-en-Artois à marseille avec des virtuoses polonais ».

37. L'Humanité, «Un très grand artiste disparait Jacques Thibaud », « Un hommage à Jacques Thibaud du grand violoniste soviétique David Ö̈strakh », L'Humanité-dimanche, «Un prince de l'archet frappé en pleine gloire. La mort de Jacques Thibaud a mis la musique en deuil », L'Humanité, « Un hommage des musiciens soviétiques à Jacques Thibaud », Les Lettres françaises, « Hommage à Jacques Thibaud », « Notre Jacques Thibaud », « Le Monde musical en deuil ». 
SLOVO

262 Les Voyages lointains des écrivains polonais $\left(\mathrm{Xx}^{\mathrm{e}}-\mathrm{XXI}^{\mathrm{e}}\right.$ siècles $)-\mathrm{n}^{\circ} 51$

Bruxelles en 1951 et Paris en 1953, étaient autant de symboles d'échanges culturels amicaux entre les peuples français et soviétiques. David Ö̈strakh l'avait décrit comme un français authentique à la radio soviétique.

1955 est également une année couronnée de succès. Les violonistes soviétiques faisaient à nouveau preuve d'un niveau remarquable au concours Marguerite Long ${ }^{38}$. Sur 29 candidats de 12 pays différents, 8 furent admis dont 3 soviétiques. Le résultat est donné dans une atmosphère houleuse, le violoniste soviétique Edouard Gratch favori du public, n'ayant obtenu que le troisième prix : Devy Erlih le premier, Olga Patkhemenko le deuxième. Valeri Klimov, soviétique également, obtint le sixième prix. Ce dernier avait été préféré à Gratch dans notre presse communiste. Sur les 67 interprètes au départ du concours de piano, les deuxième et quatrième prix de Dimitri Bachkirov, à la pénétrante musicalité, et Gleb Axelrod à la virtuosité diabolique confirmèrent la valeur des soviétiques. Le grand prix n'avait pas été attribué. Une mention spéciale est par ailleurs faite à Joulien Goutmane ${ }^{39}$. Suite aux concours, des récitals et des concerts étaient à nouveau organisés ${ }^{40}$. Toujours au piano, six soviétiques participèrent à la finale du concours Chopin de cette même année, parmi les 21 choisis $^{41}$. L'URSS était finalement représentée aux deuxième (Vladimir Ashkenazi), cinquième (Naum Starkmann), sixième (Dmitri Papyerno) et neuvième (Dmitri Sakharov) places. Par ailleurs, des concerts avaient été organisés dans toute la Pologne et payés à l'attention des candidats recalés de la première étape. Ce geste avait été mentionné deux ans auparavant, lors du Concours Wieniawski, et serait un trait caractéristique de la compréhension qui règne en ce pays pour les artistes encouragés dans leurs tâches, fussent-ils musiciens, écrivains, peintres... Le concours Ysaÿe de 1955 a également vu tous les violonistes soviétiques arriver en finale bien que le premier prix ait été décerné à l'américain

38. L'Humanité, « Ce matin s'ouvre salle Gaveau le $6^{\mathrm{e}}$ concours international Marguerite Long - Jacques Thibaud», «Le concours Marguerite Long - Jacques Thibaud », « Le grand Prix Jacques Thibaud sera décerné aujourd'hui », « Devy Erlih Grand Prix Jacques - Thibaud », Les Lettres françaises, «Le concours Jacques Thibaud-Marguerite Long », France-U.R.S.S., « Violon et piano ».

39. L'Humanité, « 27 pianistes au $2^{\mathrm{e}}$ tour du concours Marguerite Long », « Le concours Marguerite Long », « Pas de grand prix Marguerite Long. Bachkirov et Ringeissen seconds grands lauréats », « Distribution des prix Marguerite Long - Jacques Thibaud », Les Lettres françaises, « Au Concours Marguerite Long - Jacques Thibaud », France-U.R.S.S., « Violon et piano $\gg$.

40.L'Humanité, « Récital franco-soviétique salle Gaveau », « Trois concerts, six lauréats ». 41. Les Lettres françaises, « Premiers jours à Varsovie », « Le Concours Chopin à Varsovie ». 
Le rôle des virtuoses dans la valorisation de la musique soviétique. Examen de la presse communiste française

Berl Senofsky, fils de violonistes russes immigrés ${ }^{42}$. Vladimir Ashkenazi remportera quant à lui au piano, l'année suivante en 1956, le premier prix de ce même concours. Débordant le cadre de ce travail, notons que les soviétiques remporteront 15 grands prix et 69 médailles en quarante années de participation, de 1953 à $1993^{43}$.

\section{Les tournées des virtuoses non soviétiques}

Nous retrouvons bon nombre d'articles honorant de grandes vedettes non soviétiques telles Yehudi Menuhin, Efrem Kurtz, Arthur Grumiaux, Georges Enesco, Maurice Crut, Arthur Rubinstein, Fania Fénelon, Isaac Stern, Pablo Casals, Annie Fischer, Halina Stefanska, Ginette Neveu, Wanda Wikomirska, Marguerite Long ou encore Vlado Perlemuter ${ }^{44}$. Il sera systématiquement mentionné - avec une rhétorique comparable - la simplicité de leur jeu, leur discrétion, leur humilité ou encore leur fidélité vis-à-vis des textes.

On observe le lent développement des échanges entre la France et l'Union soviétique. Du côté étudiant, Michèle Boussinot, ancienne élève du conservatoire de Paris sera invitée par le conservatoire de Moscou à la demande de David Ö̈strakh ${ }^{45}$.

42. L'Humanité, « Les lauréats du Concours international de violon "Reine Élisabeth" ».

43. Frans C. Lemaire, 2004, p. 242.

44.Les Lettres françaises, «Malaise chez les musiciens», L'Humanité, « Menuhin à Paris », Ce Soir, « Yehudi Menuhin à l'Opéra », «Le Concerto en zut», « On entendra parler de Livia Rev dont la maison de la Pensée française nous a révélé le grand talent de pianiste », « Il a fallu mettre des chaises sur la scène de Pleyel pour le récital Yehudi Menuhin », « Maurice Crut, Charlie Lilamand, Ginette Guillamat », Les Lettres françaises, « Ginette Neveu », Ce Soir, « Fania Fénelon », Les Lettres françaises, « À Prades Pablo Casals qui se tait tant que parle Franco, est sorti de sa retraite pour faire entendre J.-S. Bach », L'Humanité, « Arrivée au Bourget de la grande pianiste polonaise Halina Stefanska », Ce Soir, « Festival Pablo Casals à Perpignan », « En marge du Festival de Besançon », L'Humanité, «La pianiste hongroise Annie Fischer qui jouera dimanche au Châtelet arrive aujourd'hui à Paris », «C'est à Paris, à 13 ans que Annie Fischer a donné son premier concert. Elle jouera demain au Châtelet », « Grand succès du festival de musique hongroise. Annie Fischer », Les Lettres françaises, « Encore une révélation! Annie Fischer », L'Humanité, «La célèbre pianiste hongroise Annie Fischer revient à Paris », Les Lettres françaises, «Vlado Perlemuter », L'Humanité, « La joie de vivre de Marguerite Long », «Un récital de la violoniste polonaise Wanda Wikomirska », Les Lettres françaises, « Une performance d'Arthur Rubinstein », L'Humanité, « Pablo Casals au Festival de Prades », Les Lettres françaises, « Les 80 ans de Pablo Casals », L'Humanité, «Pablo Casals : quatre-vingts ans. Cent violoncelles pour un anniversaire ».

45.L'Humanité, « Première boursière française du gouvernement soviétique la violoniste Michèle Boussinot pensionnaire du Conservatoire de Moscou », L'Humanité-dimanche, 
SLOVO

Les Voyages lointains des écrivains polonais $\left(\mathrm{XX}^{\mathrm{e}}-\mathrm{XXI}^{\mathrm{e}}\right.$ siècles $)-\mathrm{n}^{\circ} 51$

Titulaire d'une bourse d'étude qui lui a été offerte par le gouvernement soviétique, elle est la première artiste française à bénéficier d'une pareille invitation. Georges Léon dans France-U.R.S.S. se réjouissait de cette invitation, perçue comme une étape nouvelle dans les relations culturelles franco-soviétiques. Il soulignait combien cette expérience allait permettre à Michèle Boussinot de vivre «l'existence de ses camarades soviétiques », félicitant la chaleur humaine qui aurait imprégné l'enseignement musical en URSS et l'amitié qui unirait maîtres et disciples, sources du succès de tels enseignements. La francophilie personnelle d'un virtuose contribue par ailleurs à valoriser le modèle que constitue le système qui l'a formé.

Le premier virtuose à avoir été officiellement invité à Moscou, le pianiste français Samson François, donna une série de récitals en URSS et Iouri Zavadski, directeur de l'Institut théâtral l'en félicitera ${ }^{46}$. Marguerite Long et le président de la Société de musique Jacques Thibaud-Marguerite Long, le professeur Armand de Gontaut-Biron, ont été également invités par le ministère de la Culture de l'URSS ${ }^{47}$. Marguerite Long interpréta à Moscou le Concerto en sol de Ravel et la Ballade de Fauré, le public de la grande salle du Conservatoire Tchaïkovski saluant une artiste ambassadrice de la musique française, gardienne d'une grande tradition. Elle se plaît à souligner combien son voyage eut un aspect officiel ; en plein accord avec la Direction des relations culturelles du gouvernement français et pour avoir été attendue par des personnalités du ministère de la culture, l'ambassadeur Louis Joxe et des représentants du monde musical comme Gilels, Malinin, Chkolnikova ou encore Kogan. Elle fut nommée docteur Honoris Causa du conservatoire de Moscou. Sur l'initiative de l'association France-URSS, Geori Boué et Roger Bourdin interprétèrent les rôles de Tatiana et Onéguine, d'Eugène Onéguine au Bolchoï.

Les concours sont importants pour leur haute tenue artistique mais aussi pour la réunion fraternelle de tous les peuples autour de la musique. Les artistes soviétiques auraient démontré que la musique, loin d'être réservée à une prétendue élite, devrait s'adresser à ce qu'il est convenu de nommer le grand public. Ils auraient également

«Jeune femme des Épinettes Michèle Boussinot passera un an au Conservatoire de Moscou », France-U.R.S.S., « La jeune violoniste française Michèle Boussinot va travailler à Moscou avec David Ö̈strakh ».

46.France-U.R.S.S., «Le Ballet de Moscou au Châtelet », « La tournée en U.R.S.S. du pianiste Samson François ».

47.L'Humanité, « Deux musiciens français en U.R.S.S. », France-U.R.S.S., L'Humanité, «Le récital Samson François ». 
prouvé que l'art musical servirait dans de telles circonstances les efforts de ceux qui font tout pour établir entre les peuples un climat de compréhension mutuelle. La maitrise de certains artistes aurait permis de comprendre d'où venait l'excellence des méthodes d'enseignement soviétique.

Les échanges permettraient ainsi de combattre les préjugés et les images produites par certains grands journaux occidentaux, les impérialistes américains ou encore les couches les plus réactionnaires de la bourgeoisie française. Le gouvernement Laniel en est un exemple. La fonction de promotion de la culture nationale que les journalistes des Lettres françaises visent à travers leurs articles apparait bien à l'occasion de la visite annulée en 1954 des ballets soviétiques, événement qui laissera place à une indignation qui avait gagné les couches de la population ${ }^{48}$.

\section{La promotion artistique du ballet soviétique}

La danse en URSS serait restée classique et a fait l'objet d'un soutien officiel, matériel, considérable qui en aurait élargi territorialement l'audience et l'exercice ${ }^{49}$. Le style de ballet soviétique n'aurait pas été lié à une prétendue « manière russe » qui ne vaudrait que par l'éclat folklorique. La réalité contemporaine était également privilégiée $^{50}$. Aussi, insiste-t-on sur le côté positif de la vie, sur le travail, l'héroïsme et les éléments populaires. Le ballet soviétique est directement lié à l'apport des grands maîtres du passé rappelle Serge Tchoulaki : Marius Petipa, Alexandre Gorski, Michel Fokine, Lev Ivanov, qui n'auraient pas existé sans les grands compositeurs de leur temps, de même que ceux-ci n'auraient pas existé en tant qu'auteurs de ballets sans les chorégraphes cités ${ }^{51}$.

48. La Vie Ouvrière, «Enchantement des ballets soviétiques », « Les parisiens ne verront pas les ballets soviétiques ».

49. France-U.R.S.S., « Danses et ballets soviétiques ».

50. Literatournaïa Gazeta, «Le Ballet et l'Actualité », France-U.R.S.S., « Hommage aux Ballets de Moscou », « De Petipa à Oulanova, en passant par Diaghilev. La venue des ballets soviétiques continue un siècle d'échanges entre danseurs des deux pays », « Reconnaissance à "Ballet russe" », « Un retour à l'authentique », « Un enchantement qui se prolonge dans le souvenir », « Ballets soviétiques », « Opéra et ballet français au Bolchoï-Théâtre », « Les ballets soviétiques tels que je les ai vus à Moscou », « En U.R.S.S., comment naissent les étoiles ».

51.L'Humanité, « À cinq jours de la grande première à l'Opéra. Entretien avec Serge Tchoulaki Prix Staline, directeur de la troupe du ballet soviétique à Paris ». 
SLOVO

Les ballets russes sont naturellement au cœur du propos, et plus particulièrement l'homme qualifié de surprenant, généreux et providentiel que fut Diaghilev, chercheur inlassable, audacieux et voulant vivre avec son temps ${ }^{52}$. Il était aussi connu dans les milieux artistiques et intellectuels des théâtres impériaux que dans la vie sociale et politique de Saint-Pétersbourg. Diaghilev et son équipe se plaçaient au rang des plus grands novateurs, exerçant selon la tradition et transposant pour les besoins du théâtre une réalité qui leur est familière dans son essence populaire et nationale. Avec lui, on assiste à la promotion de l'origine russe du ballet moderne.

Les danseurs soviétiques font l'objet de nombreux éloges ${ }^{53}$ : parmi lesquels Eleonora Vlassova, Natalia Doudinskaïa, Vaslav Nijinski, Tamara Toumanova,

52. Les Lettres françaises, « Le souvenir de Serge de Diaguilew », « Les ballets des ChampsÉlysées », «Le Lac des cygnes », « Les Ballets Lambert », Ce Soir, « Jean Yonnel sociétaire de la Comédie-Française incarne Serge de Diaghilev au cours de la grande nuit des Ballets russes au Moulin de la Galette », Les Lettres françaises, «Le Prisonnier du Caucase », «Diaghilev était aussi critique d'art ».

53. Ce Soir, «Du clavier au stade », «Malgré bien des embûches et nombre de défection, la saison des ballets bat son plein », « Irène Strozzi va faire ses débuts dans nos studios », Regards, «Héritière de la Pavlova la danseuse de la Mort du Cygne Ludmilla Tcherina a cherché un sosie, elle a trouvé une marionnette », Ce Soir, «Si Zadig, de Pierre Petit, est trop sérieux Guignol est Pandore, de Jolivet est un badinage plein de charme », Ce Soir, «Une étoile danse au soleil », L'Humanité, « Les Parisiens pourront sans doute applaudir bientôt ces gracieuses ballerines soviétiques », « Les ballets de Léningrad à l'Opéra le 8 mai », « Les ballets soviétiques présenteront à Paris un programme très riche et varié », L'Humanité-dimanche, «En mai à l'Opéra », L'Humanité, « Cette danseuse soviétique sera dans quelques jours à Paris », « Ninel Kourgapkina dansera bientôt à l'Opéra de Paris dans "Le cavalier de bronze" », «Première arrivée à Paris de danseurs et ballerines soviétiques », « Un groupe de danseurs soviétiques arrivent ce soir au Bourget », « Les danseurs soviétiques sont depuis vendredi soir à Paris », «Roméo et Juliette répètent à l'Opéra », «Les comédiens-français qui jouaient hier "Horace" ont reçu la visite de danseurs et musiciens soviétiques », « Galina Oulanova et Lycette Darsonval se sont rencontrées à l'Opéra », « Plusieurs artistes du ballet soviétique étaient hier soir à la Comédie-Française », «Les artistes soviétiques au gala des "Amis de la danse" », «Ultimes entrechats des danseurs et danseuses soviétiques avant la grande première de lundi à l'Opéra », « Dans les salons du "continental" Galina Oulanova et le marquis de Cuevas ont sabré le champagne à l'amitié franco-soviétique », « En sortant de l'Auberge du cheval blanc Nathalia Doudinskaïa nous a parlé de la danse... sa vie », «Les étoiles de l'opéra de Paris étaient au Balcon et leurs camarades soviétiques sur la scène », « Ce soir à bureaux fermés... Premier lever de rideau sur les ballets soviétiques », «3.000 Berlinois ont applaudi les ballets soviétiques en présence de Wilhelm Pieck président de la République Démocratique Allemande », « Succès triomphal des ballets moscovites du "Berezka” à Bruxelles », « Bruxelles a pu applaudir les danseurs soviétiques », «Les comédiens-français qui jouèrent en U.R.S.S. offrent aux danseurs soviétiques deux tableaux en signe de reconnaissance et d'amitié », FranceU.R.S.S., « Nouvelle étoile de ballet », L'Humanité, « Grande danseuse de la musique française moderne Nathalie Trouhanova est morte à Moscou ». 
George Balanchine, Alla Jakovlevna Shelest, Ludmilla Tcherina, Ninel Petrova, Natacha Trouhanova, Constantin Sergueïev dont le père était ouvrier dans une usine de lampes. Des danseurs qui se distinguent moins par la virtuosité que par l'expression méditée de chaque mouvement. Ils conçoivent leur rôle en acteur, et $s$ 'attachent à en approfondir le contenu psychologique.

Galina Oulanova est décrite comme la plus grande des ballerines soviétiques et la meilleure danseuse que le monde ait connue depuis Anna Pavlova ${ }^{54}$. C'est la première ballerine de la troupe soviétique qui participait au Festival artistique de Florence en 1951. Elle participe par ailleurs à la vie sociale. À Leningrad où elle demeurait, elle était députée au Soviet local. Elle se consacrait également à l'enseignement de la chorégraphie ; créant notamment à Alma-Ata en Asie centrale, une troupe de jeunes ballerines kazakhes. Ces mérites lui valurent plusieurs prix Staline, le titre d'Artiste émérite et l'Ordre du Drapeau Rouge du Travail. La musique ne devait plus selon elle servir uniquement de support à la chorégraphie, mais plutôt posséder un contenu idéologique. La technique reste un moyen et non une fin, subordonnée à la sensibilité de l'artiste et à sa volonté de faire vivre avec intensité le personnage incarné. On trouve mentionnée son autobiographie illustrée Ballets soviétiques. Son interprétation du Roméo et Juliette de Prokofiev rappelle le véritable sens de la notion de culture européenne. La ballerine soviétique exprime la poésie de Shakespeare et l'âme du peuple soviétique, grâce à une technique élaborée au XVII ${ }^{\mathrm{e}}$ siècle par le français Beauchamp et perfectionnée par Marius Petipa, par les italiennes de Camargo et Taglioni puis les russes Fokine, Spessivtseva, Karsavina, Nijinski et Diaghilev. La venue des ballets soviétiques en France prolongeait des décennies d'échanges entre danseurs des deux pays. Lycette Darsonval disait par

54.France-U.R.S.S., «Une très grande artiste : Galina Oulanova », L'Humanité, «Galina Oulanova au festival de Florence », « La danseuse soviétique Oulanova triomphe à Florence », « La célèbre danseuse Galina Oulanova a été expulsée d'Italie par la police », L'Humanité-dimanche, « Le grand concert », L'Humanité, « À partir d'aujourd'hui Grand Concert à l'écran », «Quelques raisons du succès de Grand Concert », L'Humanité, «Un livre de Galina Oulanova paraitra à Paris en même temps qu'arrivera en France la plus illustre des ballerines soviétiques », Les Lettres françaises, «Galina Oulanova, en route pour Paris. "Comment j'ai appris mon métier..." », L'Humanité, « Galina Oulanova nous révèle les secrets de sa carrière », Les Lettres françaises, « La danse décomposée par Galina Oulanova », France-U.R.S.S., « Hommage aux Ballets de Moscou », « De Petipa à Oulanova, en passant par Diaghilev. La venue des ballets soviétiques continue un siècle d'échanges entre danseurs des deux pays », «Reconnaissance à "Ballet russe” », «Un retour à l'authentique », «Un enchantement qui se prolonge dans le souvenir », «Ballets soviétiques », « Opéra et ballet français au Bolchoï-Théâtre », «Les ballets soviétiques tels que je les ai vus à Moscou », «En U.R.S.S., comment naissent les étoiles», «Un somptueux souvenir des ballets soviétiques », « Une heure avec Oulanova ». 
SLOVO

268 Les Voyages lointains des écrivains polonais $\left(\mathrm{XX}^{\mathrm{e}}-\mathrm{XXI}^{\mathrm{e}}\right.$ siècles $)-\mathrm{n}^{\circ} 51$

exemple à propos de Galina Oulanova qu'elle avait pu retrouver en elle l'influence de l'école à laquelle elle avait elle-même été formée.

Le Festival de musique et de danses soviétiques qui s'est tenu à Paris et dans quelques villes de province en décembre 1953 fut l'occasion de dresser un riche bilan de ce que fut cette année artistique dans le cadre des rapports culturels franco-soviétiques ${ }^{55}$. 17 artistes soviétiques, musiciens, danseurs, chanteurs, marionnettiste participent du 8 au 22 décembre à 9 grands festivals. Nous observons à nouveau un rejet de l'appellation «prodige », le talent de l'artiste étant valorisé au même titre que l'excellence de l'enseignement artistique prodigué en URSS et la prospection continuellement engagée des nouveaux éléments susceptibles d'atteindre un tel niveau. Il est fait mention du grand concours de musique populaire qui devait se tenir à Moscou et des innombrables éliminatoires à travers toutes les Républiques de l'Union, réunissant une foule d'amateurs. Parmi les artistes présents, comptons Galia Izmaïlova, danseuse-étoile du théâtre Bolchoï qui avait été remarquée par la célèbre danseuse ouzbèke Tamara Khanum ou l'Ensemble de danse Piatnitski

55.L'Humanité, «Un sensationnel festival de musique et de danse soviétiques », « Igor Oistrakh sera mardi au Palais de Chaillot», L'Humanité-dimanche, « Le violoniste Ö̈strakh. La danseuse uzbek Gayla Izmailova», L'Humanité, «Trois des artistes soviétiques arrivent ce matin à Paris », «Première rencontre à Paris avec Igor Oïstrakh, Makarov et Zaria Dolhoukanova », «Igor Oïstrakh a triomphé, hier soir, au Palais de Chaillot », «Les 17 artistes soviétiques sont maintenant à Paris », « Au théâtre Marigny, la chanteuse Zaria Doloukhanova a été ovationnée hier soir », « Le Festival de danses et de musique soviétiques. Merveilleux spectacle de danses et de chants», «La voix de Zaria Doloukhanova », «Igor Oïstrakh jugé par la presse parisienne », « Rencontre au Lutétia avec les artistes soviétiques », «Zaria Doloukhanova est une reine de ce pays où le peuple est roi », L'Humanité-dimanche, «Galina Ismaïlova apporte à Paris les danses d'un Ouzbékistan qu'elle n'a pas connu », L'Humanité, « Le triomphe d'Igor Ö̈strakh à la salle Pleyel », « Samedi, avec Serge Obrastzov qui sera ce soir à la comédie-Caumartin les Parisiens ont ri du rire russe », « Ce soir, le Vel' d'Hiv' sera le cadre d'un éblouissant spectacle avec les danseurs les chanteurs et les musiciens soviétiques », « Hier soir, dans un Vél' d'Hiv' enthousiaste. Éclatant succès des artistes soviétiques », « Le magnifique récital de Bela Davidovitch au Théâtre Marigny », Les Lettres françaises, « Russes, cosaques et ouzbeks au Théâtre de Paris ou le vertige de la danse », « Voici pourquoi Doloukhanova chante si bien... », « Comment on devient tambourinaire », « Voici pourquoi Obrastzov vous fait rire... », L'Humanité, «Inoubliable soirée d'adieu des artistes soviétiques à Pleyel », « Les artistes soviétiques ont quitté la France hier soir », L'Humanité-dimanche, «Quand Serge Obratzov directeur du Théâtre des Marionnettes de Moscou parle d'art à ses collègues français », L'Humanité, «Un beau cadeau de l'inaltérable amitié! Quinze jours de chant, de danse et d'humour soviétiques », France-U.R.S.S., « Quinze jours durant danseurs, chanteurs et musiciens soviétiques ont enchanté la France ». 
(Klimov, Sorokine, Choubarine) notamment. Le parcours du tambourinaire Baraiev, originaire d'Ouzbékistan, et les recherches qu'il avait entreprises en matière folklorique seront par ailleurs mentionnées. La Radiodiffusion française s'était faite le relais de ces manifestations ${ }^{56}$.

Les représentations du ballet du Théâtre Stanislavski de Moscou au Théâtre du Châtelet, la scène originelle des ballets russes à Paris, seront abondamment commentées $^{57}$. C'est en 1918 qu'il fondait à Moscou d'abord, un studio puis un thêâtre musical (alliant chant, musique et mise en scène) qui devait fusionner, en 1940, avec celui qu'avait fondé Vladimir Nemirovitch-Dantchenko. C'était là une tentative de rénovation de la danse, un prolongement, en quelque sorte, des idées de Diaghilev. En 1941, un décret du gouvernement constituait en un organisme unique le théâtre Nemirovitch-Dantchenko et le théâtre Stanislavski. Ces deux théâtres allaient se préoccuper de créer un répertoire national correspondant aux besoins d'un public nouveau peut-on lire. L'Humanité s'y consacre tout particulièrement ${ }^{58}$. Le Maître de ballet Vladimir Bourmeister prononça l'allocution de bienvenue, soulignant quelle joie c'était de renouer avec la tradition du Châtelet.

56. L'Humanité, « Après le succès des émissions précédentes. Nouvelles retransmissions des récitals d'artistes soviétiques à la Radio les 27 et 28 janvier », « Trois récitals donnés à Paris par les artistes soviétiques seront radiodiffusés mercredi, jeudi et dimanche ».

57. Regards, « Le Lac des Cygnes retrouve Paris », Les Lettres françaises, « De la "Première" de mai 1909 à celle de juin 1956 », «Les ballets soviétiques », La Vie Ouvrière, «Les ballets soviétiques ont retrouvé Tchaikkovsky », Les Lettres françaises, «Deux ballets qui ne se ressemblent pas », France-U.R.S.S., « Le ballet de Moscou au Châtelet », Les Lettres françaises, «Ballets soviétiques ».

58. L'Humanité-dimanche, « Il y a quarante-sept ans tout juste Paris tombait amoureux des danseuses russes », L'Humanité, « Les Ballets de Moscou à Paris du 11 juin au 11 juillet », « Préparatifs pour Paris au théâtre Stanislavski de Moscou. Une interview exclusive du Maître de Ballet Vladimir Bourmeister », « Hier au foyer du Châtelet. Première rencontre entre Paris et les danseurs soviétiques », L'Humanité-dimanche, « Quarante-sept ans après... Les Ballets russes à Paris », L'Humanité, « Pleins feux sur les Étoiles soviétiques », « Première étincelante hier soir au Châtelet », «Le "Lac des cygnes" par le ballet Stanislavski », L'Humanité-dimanche, « Elles sont les élèves (lointaines) de Marius Petipa », L'Humanité, «Les danseurs soviétiques déjeunent avec les critiques parisiens », « Découverte du Ballet Soviétique avec la troupe Stanislavski », « Les ballets soviétiques au Châtelet "Les rives du bonheur" », « "Festival de ballets soviétiques", au Studio 43 », «Les Étoiles Soviétiques ont fait danser celles du Marquis de Cuevas », L'Humanité, « Les Ballets du Grand Théâtre de Moscou viendront l'an prochain à Paris... », «Fièvre à Covent Garden. Les Ballets du Grand-Théâtre de Moscou arrivent », « "Première" soviétique ce soir à Londres », «Première à Londres hier soir des ballets soviétiques », «Enthousiasme dans la presse britannique après la première représentation du ballet Bolchoï ». 
SLOVO

270 Les Voyages lointains des écrivains polonais $\left(\mathrm{XX}^{\mathrm{e}}-\mathrm{XXI}^{\mathrm{e}}\right.$ siècles $)-\mathrm{n}^{\circ} 51$

Le ballet soviétique veut que la danse soit un langage universellement compris, qui procure aux spectateurs des émotions profondes et directes. Les exemples qu'esquissaient Youri Kondratov et Alexeï Tchitchinadzé lors de répétitions sont évoqués et la jeunesse des danseurs est remarquée. Alexis Sorokine, berger sibérien orphelin très jeune, travailla aux champs, puis devint manœuvre au chemin de fer de Novossibirsk. Sa vocation surgit le jour où il entra comme machiniste dans un théâtre. Il suivit des cours de danse, puis fut engagé au Théâtre de Saratov.

Comme les instrumentistes nous observons les éloges faits à l'égard de troupes ou de danseurs non soviétiques : les ballets de Monte-Carlo dirigés par le Marquis de Cuevas et son maître John Taras ${ }^{59}$, le ballet d'Elsa Triolet ${ }^{60}$ qui a eu le courage et le mérite d'introduire dans les Ballets des Champs-Élysées les amours fraîches et pures de jeunes ouvriers, le Sadler's Wells Ballet ${ }^{61}$, les Ballets de l'Étoile ${ }^{62}$, Maurice Béjart ${ }^{63}$ dont la science est exemptée de faits spectaculaires, Roland Petit ${ }^{64}$, Yvette Chauviré ${ }^{65}$, Irène Strozzi ${ }^{66}$, Janine Charrat $^{67}$, Françoise Adret ${ }^{68}$ qui à

59. Les Lettres françaises, « On dansera ce soir à la République et aux Champs-Élysées », «Les ballets de Monte-Carlo à l'Alhambra », «Le Ballet de Monte-Carlo», «Les Théâtres nationaux », « Du "Tricorne" au "Peintre et son modèle". Un pas à côté plutôt qu'un pas en avant», Regards, «Aux ballets de Monte-Carlo. On parle des langues différentes mais on danse d'un même cœur », L'Humanité-dimanche, « Le Grand Ballet du Marquis de Cuevas », L'Humanité, « Festival Tchaikovsky pour la rentrée du Ballet de Cuevas », Les Lettres françaises, « La Damnée de Walter Gore », « Traditionalisme contre tradition », L'Humanité-dimanche, « La semaine culturelle. La création chorégraphique avec : Janine Monin, Igor Fosca, Youra Loboff, Gayle Spear gala présenté par les J.M.F. ».

60.L'Humanité-dimanche, «Antoine et Antoinette dansent aux ballets des ChampsÉlysées », Regards, « Le ballet d'Elsa Triolet ».

61.Regards, «The Sadlers Wells Ballet vient danser à Paris », Ce Soir, « La danse en Angleterre », « Les Ballets du “Sadler's Wells" », « Une Troupe d'une cohésion parfaite : le “Sadler's Wells Ballet” », Les Lettres françaises, « Le Sadler's Wells Ballet à l'Opéra ».

62.L'Humanité, «Un éventail de bonne humeur. Les ballets de l'Étoile », « À l'Étoile et aux Champs-Élysées. Ballets ».

63. Les Lettres françaises, « Le Gala de la danse ».

64. Regards, « Roland Petit va danser "Guernica" », Ce Soir, « "La Croqueuse de diamants" à Marigny $\gg$.

65.L'Humanité, « Yvette Chauviré et "Les Ballets de Marigny” ».

66. Ce Soir, «Une étoile danse au soleil ».

67. Ce Soir, « On peut enfin entendre de grands orchestres symphoniques en juillet à Paris grâce aux "Nuits Musicales" de Chaillot », L'Humanité, « Les ballets de Janine Charrat à l'Empire ».

68. Ce Soir, « Toute la danse était représentée hier soir au Palais de Chaillot ». 
l'occasion de la représentation de Terrain Vague aurait prouvé qu'en maintenant la danse classique dans ses solides traditions, les thèmes de l'époque étaient en mesure de lui apporter un sang nouveau, Mathilde Roelens et Paul Dougnac (dont on s'attardait sur le parcours d'ouvrier chez Citroën $)^{69}$, ou encore Katherine Dunham ${ }^{70}$, félicitée après le remaniement express d'un spectacle jugé trop proche du music-hall hollywoodien. On observe à nouveau, avec le ballet, combien toutes les orientations induites par le réalisme socialiste pouvaient s'appréhender avec notre presse communiste de l'époque (que les articles fussent en liens directs ou a priori exempts de références à l'Union soviétique), cette dernière s'étant appropriée les critères d'appréciation du régime soviétique.

\section{Le rôle du cinéma soviétique dans la valorisation des virtuoses}

Le cinéma a enfin été un vecteur important de la réception en France de la musique et du ballet soviétique. Le documentaire Le Grand Concert réalisé en 1951 par Vera Stroeva montrerait la liaison indissoluble qui existe entre le peuple soviétique et ses artistes ${ }^{71}$. On assiste à l'interpénétration de la ville et de la campagne (la venue des kolkhoziens à l'Opéra où a lieu une représentation du Prince Igor, la visite des artistes au kolkhoze «Victoire » où les paysans leur offrent un banquet, chantent et dansent pour eux - c'est l'atmosphère des Cosaques du Kouban que nous retrouverions là -, l'admission au conservatoire de Moscou des jeunes paysans doués) qui contribue à unifier l'Union soviétique. Le cinéma soviétique triomphe en 1953 et 1954 sur les écrans parisiens avec Le Grand Concert justement, qui, au cours des cinq premières semaines d'exclusivité avait été vu par 80000 spectateurs $^{72}$. Le film de Vsevolod Poudovkine La Moisson sorti en 1953 et Le Grand Concert

69. Les Lettres françaises, « $\grave{A}$ la recherche des voies nouvelles de la musique et de la danse », L'Humanité-dimanche, « "La danse au service du peuple" mot d'ordre de Paul et Mathilde Dougnac et de toute leur activité ».

70.L'Humanité-dimanche, « Katherine Dunham fait sa rentrée au Théâtre des ChampsÉlysées », « Pour satisfaire le public parisien Katherine Dunham a remanié son spectacle en un temps record $\gg$.

71. Ce Soir, «Un merveilleux cadeau de Noël : Le Grand Concert ou les splendeurs de l'Opéra de Moscou », « "Le Grand Concert" ou l'enchantement de la Musique », Les Lettres françaises, « Le Grand Concert. Deux heures à l'Opéra de Moscou », « Le Grand Concert ».

72.France-U.R.S.S., « Le "Grand Concert" : 80.000 spectateurs en cinq semaines à Paris », « La Moisson et le Grand Concert entreprennent leur “Tour de France” ». 
SLOVO

272 Les Voyages lointains des écrivains polonais $\left(\mathrm{Xx}^{\mathrm{e}}-\mathrm{XXI}^{\mathrm{e}}\right.$ siècles $)-\mathrm{n}^{\circ} 51$

seront alors par ailleurs présentés pour la première fois dans de nombreuses villes de province grâce aux comités France-URSS

Le film d'Ivanovski et Rappaport Le Concert des Maîtres de l'Art, en fait sous-titre de Étoiles du ballet russe joua également un rôle prépondérant ${ }^{73}$. Sorti en 1954, il met à l'honneur le jeune cinéma ukrainien avec un panorama de la vie artistique en URSS, allant de Tchaïkovski à Chostakovitch où ballets, symphonies, opéras, chants et danses populaires se mêlent et alternent. Le génie de Galina Oulanova est remarqué par exemple. Pour ses zélateurs, le film aiderait à mieux comprendre les conditions historiques dans lesquelles furent jadis conçus certains chefs-d'œuvre qui constituaient l'héritage classique et national des créateurs soviétiques. Le Festival de Cannes de 1954 met à l'honneur ce documentaire ${ }^{74}$. Ce film, parce qu'il suivait les récentes tournées d'artistes soviétiques en France, préludant à la série de représentations que donnèrent à l'Opéra de Paris quelques-uns de ses protagonistes, était susceptible de préparer des échanges culturels entre la France et l'URSS.

\section{Conclusion}

Cette étude, plutôt que d'éclairer la réception intellectuelle - ou spécialisée du concept de réalisme socialiste par l'opinion publique, privilégie l'expérience ordinaire, par la population, des prestations des virtuoses soviétiques. Nous avons fait le choix de reconstituer un discours général de cette presse communiste française, englobant tous les articles recensés qui traduisent l'essor culturel et social de l'URSS. Cette approche neuve connecte la musicologie à des disciplines telles l'esthétique et les sciences politiques principalement. Les actualités traitées et les propos sur les personnalités étaient donc adaptés par les sympathisants pour véhiculer le plus directement possible les préceptes réalistes en musique. La propagande soviétique se voit par ailleurs transposée à une actualité sans

73. Les Lettres françaises, « Le Concert des Maîtres de l'Art russe », L'Humanité, « Ces étoiles du ballet russe seront au Festival de Cannes », «En avril, sur les écrans parisiens. En mai, sur la scène de l'Opéra. Les ballets soviétiques », L'Humanité-dimanche, «Les Maîtres du ballet russe passe dans une salle parisienne », L'Humanité, «Un des grands succès du Festival de Cannes 1954: "Étoiles de ballets russes" », « Ce soir, programme unique à Pleyel: "Étoiles de ballets russes" le film de Cannes qui réunit les plus beaux spectacles applaudis à Moscou et Léningrad par les Comédiens-Français », La Nouvelle Critique, «"Le Concert des Maîtres de l'art” », L’Humanité-dimanche, «La dernière répétition des Ballets Soviétiques », L'Humanité, «Étoiles du Ballet Russe ».

74. Les Lettres françaises, « Les Étoiles du ballet russe », France-U.R.S.S., «Étoiles du ballet russe », Les Lettres françaises, « Étoiles des ballets russes ». 
lien direct avec la musique soviétique. Il conviendrait pour ouvrir le débat de connaître les répertoires de tels concours internationaux et les décisionnaires des œuvres imposées. Comment un virtuose aurait pu servir les idéaux du Parti avec l'interprétation d'œuvres imposées qui n'auraient pas reçu l'adhésion du Parti par exemple?

\section{Bibliographie}

\section{Ce Soir}

Ce Soir, 12 octobre 1944, « Yehudi Menuhin à l'Opéra », Jean Wiéner.

Ce Soir, 9 mars 1945, « La danse en Angleterre », René Luquet.

Ce Soir, 16 mars 1945, « Les Ballets du “Sadler's Wells” », René Luquet.

Ce Soir, 25 juillet 1946, «Du clavier au stade », Lucile Augeron ; « Malgré bien des embûches et nombre de défections, la saison des ballets bat son plein », A. Richard Balducci.

Ce Soir, 15 août 1946, «Irène Strozzi va faire ses débuts dans nos studios », A. Richard Balducci.

CeSoir, 8 juillet 1948, « On entendra parler de Livia Rev dont la maison de la Pensée française nous a révélé le grand talent de pianiste », Hélène Jourdan-Morhange.

Ce Soir, 15 juillet 1948, «Grande soirée de gala à l'Opéra », Hélène Jourdan-Morhange.

Ce Soir, 30 septembre 1948, «Une troupe d'une cohésion parfaite: le "Sadler's Wells Ballet” », Hélène Jourdan-Morhange.

Ce Soir, 28 octobre 1948, « Il a fallu mettre des chaises sur la scène de Pleyel pour le récital Yehudi Menuhin », Hélène Jourdan-Morhange.

Ce Soir, 30 avril 1949, « Une étoile danse au soleil ».

Ce Soir, 9 juin 1949, « Maurice Crut, Charlie Lilamand, Ginette Guillamat », Hélène Jourdan-Morhange.

Ce Soir, 14 juillet 1949, «On peut enfin entendre de grands orchestres symphoniques en juillet à Paris grâce aux "Nuits Musicales" de Chaillot », Hélène Jourdan-Morhange.

Ce Soir, 9 mars 1950, « Fania Fénelon », Hélène Jourdan-Morhange.

Ce Soir, 28 septembre 1950, « "La Croqueuse de diamants" à Marigny », Hélène Jourdan-Morhange.

Ce Soir, 2 novembre 1950, « Milstein l'invincible », Hélène Jourdan-Morhange.

CeSoir, 16 novembre 1950, « Jascha Horenstein à Paris », Hélène Jourdan-Morhange. 
SLOVO

274 Les Voyages lointains des écrivains polonais $\left(\mathrm{XX}^{\mathrm{e}}-\mathrm{XXI}^{\mathrm{e}}\right.$ siècles $)-\mathrm{n}^{\circ} 51$

Ce Soir, 15 décembre 1950, « Pour la première fois depuis de nombreuses années, deux grands virtuoses soviétiques, L. Oborine (pianiste) et I. Bezrodni (violoniste) sont à Paris où ils joueront dimanche à Pleyel », Marcel Besset.

Ce Soir, 4 janvier 1951, « Léo Oborine et Igor Bezrodni au Palais de Chaillot », Hélène Jourdan-Morhange.

Ce Soir, 10 mai 1951, «Jean Yonnel sociétaire de la Comédie-Française incarne Serge de Diaghilev au cours de la grande nuit des Ballets russes au Moulin de la Galette », Marcel Frère.

Ce Soir, 23 juin 1951, «Toute la danse était représentée hier soir au Palais de Chaillot », Marcel Frère.

Ce Soir, 30 juin 1951, « Des virtuoses du monde entier se sont retrouvés à Bruxelles ».

Ce Soir, 27 juillet 1951, « Festival Pablo Casals à Perpignan », Hélène Jourdan-Morhange.

Ce Soir, 20 septembre 1951, « En marge du Festival deBesançon », Hélène Jourdan-Morhange.

Ce Soir, 6 décembre 1952, « Le disque que vous pouvez offrir pour les étrennes: Capriccio espagnol de Rimski-Korsakov, Fantaisie sur des thèmes de “Carmen” », Paul Grangeon.

Ce Soir, 18 décembre 1952, « La violoniste Blanche Tarjus obtient le troisième prix international Wieniawski », Hélène Jourdan-Morhange.

Ce Soir, 19 décembre 1952, «Un merveilleux cadeau de Noël : Le Grand Concert ou les splendeurs de l'Opéra de Moscou », Janine Bouissounouse.

\section{Ce Soir dimanche}

Ce Soir dimanche, 22 janvier 1947, « Le Concerto en zut », André Wurmser.

Ce Soir dimanche, 20 décembre 1950, «À Pleyel: Oborine et Bezrodni deux (très) grands virtuoses soviétiques », Hélène Jourdan-Morhange.

Ce Soir dimanche, 8, 9 juillet 1951, «15 ans. Gérard Jarry (Prix Marguerite Long Jacques Thibaud) sera dans quelques années, grâce à son travail l'un des grands noms de la musique », R. L.

Ce Soir dimanche, 24 décembre 1952, « "Le Grand Concert” ou l'enchantement de la Musique », Louis Durey.

\section{Chroniques étrangères}

Chroniques étrangères. U.R.S.S., 30 novembre 1954, « La saison théâtrale et musicale de Moscou $\gg$.

\section{France-U.R.S.S.}

France-U.R.S.S., mai 1947, «D Danses et ballets soviétiques », Yves Bonnat. France-U.R.S.S., juillet 1950, «Une très grande artiste : Galina Oulanova ». France-U.R.S.S., septembre 1950, «Pour la paix, le peuple soviétique répond: présent! ». 
France-U.R.S.S., février 1951, « L. Oborine et I. Bezrodni emportent du public français un chaleureux souvenir $\gg$.

France-U.R.S.S., mars 1953, «Le "Grand Concert": 80.000 spectateurs en cinq semaines à Paris $\gg$, Jacques Sylvain.

France-U.R.S.S., juillet-août 1953, «Une violoniste de 24 ans a ébloui Paris qui pourtant ne se laisse pas séduire facilement $\gg$.

France-U.R.S.S., septembre 1953, «Les violonistes soviétiques l'ont prouvé : la musique passionne "le grand public" », Georges Léon.

France-U.R.S.S., février 1954, «Quinze jours durant danseurs, chanteurs et musiciens soviétiques ont enchanté la France », Georges Léon.

France-U.R.S.S., mars 1954, «Un grand pianiste soviétique à Paris ».

France-U.R.S.S., avril 1954, «Ivan Petrov dans Boris Godounov à l'Opéra », Georges Léon.

France-U.R.S.S., mai 1954, «Hommage aux Ballets de Moscou », Weill Hallé ; «De Petipa à Oulanova, en passant par Diaghilev», «Reconnaissance à "Ballet russe" », Jean Cocteau ; « Un retour à l'authentique », Fernand Gregh ; «Un enchantement qui se prolonge dans le souvenir», Gérard Bauer; «Ballets soviétiques », Galina Oulanova; «Opéra et ballet français au Bolchoï-Théâtre », « Les ballets soviétiques tels que je les ai vus à Moscou », Pierre Debray ; « En U.R.S.S., comment naissent les étoiles », Georges Léon.

France-U.R.S.S., juin 1954, «Un somptueux souvenir des ballets soviétiques », « Étoiles du ballet russe », Jacques Doniol Valcroze.

France-U.R.S.S., octobre 1954, « La Moisson et le Grand Concert entreprennent leur "Tour de France" ».

France-U.R.S.S., décembre 1954, « Le Concerto de Brahms par David Ö̈strakh », Georges Léon ; «La jeune violoniste française Michèle Boussinot va travailler à Moscou avec David Ö̈strakh », Georges Léon.

France-U.R.S.S., avril 1955, « Le violoniste soviétique Léonide Kogan en France », Georges Léon.

France-U.R.S.S., mai 1955, « Deux musiciens français en U.R.S.S. ».

France-U.R.S.S., août 1955, «Violon et piano », Georges Léon.

France-U.R.S.S., novembre 1955, « Nouvelle étoile de ballet ».

France-U.R.S.S., décembre 1955, «Une heure avec Oulanova », Jacques Dubois.

France-U.R.S.S., juillet 1956, «Le ballet de Moscou au Châtelet », Victoria Achères ; «La tournée en U.R.S.S. du pianiste Samson François », Georges Léon.

France-U.R.S.S., décembre 1956.

\section{Izvestia}

Izvestia, 2 avril 1937. 
SLOVO

276 Les Voyages lointains des écrivains polonais $\left(\mathrm{XX}^{\mathrm{e}}-\mathrm{XXI}^{\mathrm{e}}\right.$ siècles $)-\mathrm{n}^{\circ} 51$

Izvestia, 3 avril 1937, « Le triomphe des musiciens soviétiques ».

Izvestia, 15 juin 1951.

\section{L'Humanité}

L'Humanité, 8 et 9 octobre 1944, « Menuhin à Paris ».

L'Humanité, 10 avril 1950, «Nijinski qui fut le "meilleur danseur du monde" est mort à Londres ».

L'Humanité, 15 décembre 1950, « Dimanche soir à Pleyel Léon Oborine Igor Bezrodni merveilleux musiciens ».

L'Humanité, 22 décembre 1950, « Le jeu de haute qualité des virtuoses soviétiques », Serge Nigg.

L'Humanité, 8 mai 1951, « Galina Oulanova au festival de Florence ».

L'Humanité, 25 mai 1951, «Un violoniste soviétique remporte le "Grand Prix musical de la Reine Élisabeth de Belgique” ».

L'Humanité, 30 mai 1951, «Arrivée au Bourget de la grande pianiste polonaise Halina Stefanska ».

L'Humanité, 5 juin 1951.

L'Humanité, 16 juin 1951, «La danseuse soviétique Oulanova triomphe à Florence $\gg$.

L'Humanité, 2 août 1951, « La célèbre danseuse Galina Oulanova a été expulsée d'Italie par la police $\gg$.

L'Humanité, 4 février 1952, «Les ballets de Janine Charrat à l'Empire », Gilbert Bloch.

L'Humanité, 27 juin 1952.

L'Humanité, 30 juin 1952, «Yvette Chauviré et "Les Ballets de Marigny" », Gilbert Bloch.

L'Humanité, 17 décembre 1952, « À partir d'aujourd'hui Grand Concert à l'écran ».

L'Humanité, 18 décembre 1952, « Igor Ö̈strakh remporte le concours de violon de Poznan. Blanche Tarjus 3' ».

L'Humanité, 7 janvier 1953, «Quelques raisons du succès de Grand Concert », Gilbert Bloch.

L'Humanité, 30 mai 1953 « Neuf musiciens soviétiques bientôt à Paris ? ».

L'Humanité, 9 juin 1953, « Huit musiciens soviétiques à Paris », « David Oistrakh et Lev Oborine à Paris ».

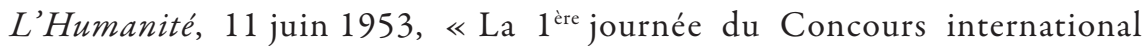
Marguerite Long - Jacques Thibaud. Le jeune violoniste soviétique R. Sobolevski a fait une grande impression sur le public », Georges Léon.

L'Humanité, 12 juin 1953, « $2^{\text {ème }}$ Journée de concours salle Gaveau », Georges Léon. 
L'Humanité, 13 juin 1953, « Le concours Long-Thibaud. Finale disputée aujourd'hui par 8 sélectionnés dont Sobolevsky et N. Chkolnikova », Georges Léon.

L'Humanité, 15 juin 1953, «Triomphal succès des musiciens soviétiques au concours Long-Thibaud », Georges Léon.

L'Humanité, 16 juin 1953, «Les commentaires de la presse sur le triomphal succès de Nelly Chkolnikova », «Quelques raisons des succès des jeunes musiciens soviétiques », Gilbert Bloch; « Les pianistes s'affrontent à leur tour salle Gaveau ». L'Humanité, 17 juin 1953, « 40 pianistes restent en ligne au concours Marguerite-Long 》.

L'Humanité, 19 juin 1953, «Pour "L'Humanité" David Oïstrakh évoque sa vie quotidienne », «Avant les deux concerts de Chaillot Kabalevski, Ö̈strakh et Oborine au C.N.E. », «Au concours Marguerite Long remous dans le public après la décision du jury ».

L'Humanité, 22 juin 1953, « Finale mouvementée au concours Marguerite Long Jacques Thibaud », Georges Léon.

L'Humanité, 25 juin 1953, «Le 29 juin à Chaillot avec David Ö̈strakh Lev Oborine un très grand pianiste », « Dimanche, à Pleyel $2^{\mathrm{e}}$ concert avec les lauréats du concours Long-Thibaud ».

L'Humanité, 27 juin 1953, « Triomphal succès de David Ö̈strakh à Chaillot ».

L'Humanité, 29 juin 1953, « Le triomphal concert. David Ö̈strakh au Palais de Chaillot », Georges Léon.

L'Humanité, 30 juin 1953, «La presse unanime rend hommage au talent de David Ö̈strakh », «Nouveau triomphal succès hier soir à Chaillot. David Ö̈strakh et Lev Oborine ».

L'Humanité, $1^{\text {er }}$ juillet 1953, «Après leur triomphe à Chaillot David Ö̈strakh et Lev Oborine joueront ce soir à Lille. Dernier concert à Pleyel le 3 juillet », «Les jeunes virtuoses soviétiques à Bordeaux ».

L'Humanité, 3 juillet 1953, « David Ö̈strakh et Lev Oborine à l'Opéra de Lille ». L'Humanité, 8 juillet 1953, « Les adieux de Lev Oborine et de David Oistrakh ». L'Humanité, 19 août 1953, « David Oistrakh "artiste du peuple” ».

L'Humanité, 3 septembre 1953, « Un très grand artiste disparait Jacques Thibaud », Georges Soria.

L'Humanité, 5 septembre 1953, «Un hommage à Jacques Thibaud du grand violoniste soviétique David Oïstrakh ».

L'Humanité, 8 septembre 1953, «Un hommage des musiciens soviétiques à Jacques Thibaud ».

L'Humanité, 31 octobre 1953, «Festival Tchaikovsky pour la rentrée du Ballet de Cuevas », Gilbert Bloch. 
SLOVO

278 Les Voyages lointains des écrivains polonais $\left(\mathrm{XX}^{\mathrm{e}}-\mathrm{XXI}^{\mathrm{e}}\right.$ siècles $)-\mathrm{n}^{\circ} 51$

L'Humanité, 2 décembre 1953, « Un sensationnel festival de musique et de danse soviétiques 》.

L'Humanité, 5 décembre 1953, « Igor Ö̈strakh sera mardi au Palais de Chaillot ». L'Humanité, 7 décembre 1953, « Prestiges et vertus du virtuose », Gilbert Bloch ;

«Trois des artistes soviétiques arrivent ce matin à Paris ».

L'Humanité, 8 décembre 1953, « Première rencontre à Paris avec Igor Ö̈strakh, Makarov et Zaria Dolhoukanova », Georges Léon.

L'Humanité, 9 décembre 1953, «Igor Ö̈strakh a triomphé, hier soir, au Palais de Chaillot », Georges Léon; «Les 17 artistes soviétiques sont maintenant à Paris ».

L'Humanité, 10 décembre 1953, «Au Théâtre Marigny, la chanteuse Zaria Doloukhanova a été ovationnée hier soir », Georges Léon.

L'Humanité, 11 décembre 1953, « Le Festival de danses et de musique soviétiques. Merveilleux spectacle de danses et de chants », Simone Tery; «La voix de Zaria Doloukhanova », Georges Léon; «Igor Ö̈strakh jugé par la presse parisienne ».

L'Humanité, 12 décembre 1953, « Zaria Doloukhanova est une reine de ce pays où le peuple est roi $\gg$, Simone Tery.

L'Humanité, 14 décembre 1953, «Rencontre au Lutétia avec les artistes soviétiques », « Le triomphe d'Igor Ö̈strakh à la salle Pleyel », Georges Léon.

L'Humanité, 15 décembre 1953, « Samedi, avec Serge Obrastzov qui sera ce soir à la Comédie-Caumartin les Parisiens ont ri du rire russe », Simon Tery.

L'Humanité, 16 décembre 1953, «Ce soir, le Vel' d'Hiv' sera le cadre d'un éblouissant spectacle avec les danseurs les chanteurs et les musiciens soviétiques $\gg$.

L'Humanité, 17 décembre 1953, « Hier soir, dans un Vél' d'Hiv' enthousiaste. Éclatant succès des artistes soviétiques », Simone Tery ; « Le magnifique récital de Bela Davidovitch au Théâtre Marigny », Georges Léon.

L'Humanité, 23 décembre 1953, «Les marionnettistes français reçoivent Serge Obrastzov ».

L'Humanité, 25 décembre 1953, « Les marionnettes de Serge Obrastzov et celles d'Yves Joly se sont rencontrées à la maison de la Chimie », « Inoubliable soirée d'adieu des artistes soviétiques à Pleyel $\gg$.

L'Humanité, 26 décembre 1953, « Les artistes soviétiques ont quitté la France hier soir ».

L'Humanité, 28 décembre 1953, «Un beau cadeau de l'inaltérable amitié ! Quinze jours de chant, de danse et d'humour soviétiques », Gilbert Bloch.

L'Humanité, 6 janvier 1954, «La pianiste hongroise Annie Fischer qui jouera dimanche au Châtelet arrive aujourd'hui à Paris », Georges Léon. 
L'Humanité, 8 janvier 1954, «Nelly Chkolnikova qui conquit Paris cet été triomphe à Moscou », « Nouvelles retransmissions des récitals d'artistes soviétiques à la Radio les 27 et 28 janvier ».

L'Humanité, 9 janvier 1954, « C'est à Paris, à 13 ans que Annie Fischer a donné son premier concert. Elle jouera demain au Châtelet », Georges Léon.

L'Humanité, 11 janvier 1954, « Grand succès du festival de musique hongroise. Annie Fischer », Georges Léon.

L'Humanité, 19 janvier 1954, «Les Parisiens pourront sans doute applaudir bientôt ces gracieuses ballerines soviétiques ».

L'Humanité, 26 janvier 1954, «Trois récitals donnés à Paris par les artistes soviétiques seront radiodiffusés mercredi, jeudi et dimanche ».

L'Humanité, 27 janvier 1954, « Les ballets de Léningrad à l'Opéra le 8 mai ».

L'Humanité, 3 février 1954, « Un des plus grands pianistes soviétiques vient à Paris dans quelques jours $\gg$.

L'Humanité, 6 février 1954, « Le grand pianiste soviétique Émile Guillels qui sera dans quelques jours à Paris remporta son premier triomphe à 15 ans ».

L'Humanité, 11 février 1954, « Le grand pianiste soviétique Émile Guillels est arrivé hier à Paris », Georges Léon.

L'Humanité, 13 février 1954, «Avant son concert de demain salle Pleyel Émile Guillels évoque pour vous quelques souvenirs parisiens », Georges Léon.

L'Humanité, 15 février 1954, « Hier soir, salle Pleyel Grand succès du récital Émile Guillels », Georges Léon.

L'Humanité, 18 février 1954, « La célèbre pianiste hongroise Annie Fischer revient à Paris ».

L'Humanité, 19 février 1954, « Émile Guillels, Michèle Boussinot, Aimé Doniat participeront dimanche à Pleyel à l'hommage à Stalingrad », F. G.

L'Humanité, 20 février 1954, «Un très grand artiste: Émile Guillels fait l'unanimité de la presse parisienne ».

L'Humanité, 24 février 1954, «Émile Guillels donnera un récital d'œuvres soviétiques modernes jusqu'à présent jamais exécutées en France ».

L'Humanité, 27 février 1954, «Immense succès du récital d'Émile Guillels à la salle Pleyel ».

L'Humanité, $1^{\text {er }}$ mars 1954, «Émile Guillels (magicien du piano) a connu à Pleyel un nouveau triomphe $\gg$, Gilbert Bloch.

L'Humanité, 3 mars 1954, « Le chanteur soviétique Ivan Petrov est arrivé à Paris ».

L'Humanité, 4 mars 1954, « Ivan Petrov à l'Opéra ».

L'Humanité, 5 mars 1954, «Ivan Petrov deux fois Prix Staline à 34 ans sera Boris Godounov ».

L'Humanité, 6 mars 1954, « Ivan Petrov triomphe à l'Opéra dans "Boris Godounov" ». 
SLOVO

280 Les Voyages lointains des écrivains polonais $\left(\mathrm{XX}^{\mathrm{e}}-\mathrm{XXI}^{\mathrm{e}}\right.$ siècles $)-\mathrm{n}^{\circ} 51$

L'Humanité, 8 mars 1954, «Ivan Petrov inoubliable Boris Godounov », Gilbert Bloch ; « Quelques opinions de la critique sur Ivan Petrov ».

L'Humanité, 11 mars 1954, «Hier, réception à l'Opéra en l'honneur d'Ivan Petrov », « Dernier concert d'Émile Guillels mardi à Chaillot ».

L'Humanité, 15 mars 1954, « Ivan Petrov incomparable Méphisto à l'Opéra », Gilbert Bloch.

L'Humanité, 16 mars 1954, «Brillante réception en l'honneur des artistes soviétiques 》.

L'Humanité, 20 mars 1954, « Ces étoiles du ballet russe seront au Festival de Cannes ».

L'Humanité, 22 mars 1954, « Nelly Chkolnikova et Evgeny Malinine joueront demain soir au théâtre des Champs-Élysées ».

L'Humanité, 24 mars 1954, «Immense succès d'Ivan Petrov au Palais de Chaillot $\gg$.

L'Humanité, 25 mars 1954, « Ivan Petrov artiste soviétique », Gilbert Bloch.

L'Humanité, 26 mars 1954, «Un émouvant et magnifique concert: celui de Nelly Chkolnikova et Evgeny Malinine au théâtre des Champs-Élysées », Georges Léon.

L'Humanité, 29 mars 1954, «Les ballets soviétiques présenteront à Paris un programme très riche et varié $\gg$.

L'Humanité, 31 mars 1954, «Découverte d'“Eugène Onéguine” » opéra favori des Soviétiques... et inconnu des Français », Gilbert Bloch.

L'Humanité, 2 avril 1954, «En attendant le concert de Nelly Chkolnikova », « En avril, sur les écrans parisiens. En mai, sur la scène de l'Opéra. Les ballets soviétiques ».

L'Humanité, 5 avril 1954, «Dernier récital de Nelly Chkolnikova aux Champs-Élysées », Georges Léon.

L'Humanité, 21 avril 1954, « Un des grands succès du Festival de Cannes 1954: "Étoiles de ballets russes" ».

L'Humanité, 22 avril 1954, «Ce soir, programme unique à Pleyel : "Étoiles de ballets russes" le film de Cannes qui réunit les plus beaux spectacles applaudis à Moscou et Léningrad par les Comédiens-Français », R. B.

L'Humanité, 24 avril 1954, « Cette danseuse soviétique sera dans quelques jours à Paris ».

L'Humanité, 27 avril 1954, « Ninel Kourgapkina dansera bientôt à l'Opéra de Paris dans "Le cavalier de bronze" ».

L'Humanité, 28 avril 1954, « Première arrivée à Paris de danseurs et ballerines soviétiques ». 
L'Humanité, 29 avril 1954, «Un livre de Galina Oulanova paraitra à Paris en même temps qu'arrivera en France la plus illustre des ballerines soviétiques ».

L'Humanité, 30 avril 1954, « Un groupe de danseurs soviétiques arrivent ce soir au Bourget $\gg$.

L'Humanité, 3 mai 1954, « Les danseurs soviétiques sont depuis vendredi soir à Paris ».

L'Humanité, 4 mai 1954, «Roméo et Juliette répètent à l'Opéra », « À cinq jours de la grande première à l'Opéra. Entretien avec Serge Tchoulaki », Georges Léon.

L'Humanité, 5 mai 1954, «Galina Oulanova nous révèle les secrets de sa carrière », Gilbert Bloch, « Les comédiens-français qui jouaient hier "Horace" ont reçu la visite de danseurs et musiciens soviétiques », « Galina Oulanova et Lycette Darsonval se sont rencontrées à l'Opéra », « Plusieurs artistes du ballet soviétique étaient hier soir à la Comédie-Française », « Les artistes soviétiques au gala des "Amis de la danse" »,

L'Humanité, 6 mai 1954, «Ultimes entrechats des danseurs et danseuses soviétiques avant la grande première de lundi à l'Opéra », « Dans les salons du "continental" Galina Oulanova et le marquis de Cuevas ont sablé le champagne à l'amitié franco-soviétique ».

L'Humanité, 7 mai 1954, « Michel Tchoulaki directeur des ballets soviétiques à Paris : cette photo amuserait bien les habitants de Moscou », « En sortant de l'Auberge du cheval blanc Nathalia Doudinskaïa nous a parlé de la danse... sa vie », Georges Léon.

L’Humanité, 8 mai 1954, « Les étoiles de l'opéra de Paris étaient au Balcon et leurs camarades soviétiques sur la scène ».

L'Humanité, 10 mai 1954, « Ce soir à bureaux fermés... Premier lever de rideau sur les ballets soviétiques », Georges Léon.

L'Humanité, 19 mai 1954, « Suivez le guide... de l'Académie Charles-Cros ».

L'Humanité, 29 mai 1954, «3.000 Berlinois ont applaudi les ballets soviétiques en présence de Wilhelm Pieck président de la République démocratique allemande ».

L'Humanité, 23 juin 1954, « Bruxelles a pu applaudir les danseurs soviétiques ».

L'Humanité, 24 août 1954, « Le grand pianiste soviétique Émile Guillels viendra le mois prochain à Besançon ».

L'Humanité, 20 septembre 1954, « Les comédiens-français qui jouèrent en U.R.S.S. offrent aux danseurs soviétiques deux tableaux en signe de reconnaissance et d'amitié ».

L'Humanité, 2 novembre 1954, «Étoiles du Ballet Russe », Georges Léon. 
SLOVO

282 Les Voyages lointains des écrivains polonais $\left(\mathrm{XX}^{\mathrm{e}}-\mathrm{XXI}^{\mathrm{e}}\right.$ siècles $)-\mathrm{n}^{0} 51$

L'Humanité, 15 novembre 1954, «Première boursière française du gouvernement soviétique la violoniste Michèle Boussinot pensionnaire du Conservatoire de Moscou ».

L'Humanité, 20 novembre 1954, «Succès triomphal des ballets moscovites du "Berezka" à Bruxelles ».

L'Humanité, 23 novembre 1954, « David Oïstrakh : "Le rideau de fer est américain” ». L'Humanité, 24 novembre 1954, «Le pianiste soviétique Émile Guillels est à nouveau l'hôte de Paris ».

L'Humanité, 25 novembre 1954, «Nina Emilianova jouera ce soir au Conservatoire de Paris $\gg$.

L'Humanité, 3 décembre 1954, « Émile Guillels et André Cluytens ».

L'Humanité, 2 février 1955, « Le grand violoniste soviétique Léonide Kogan sera pour la première fois à Paris le 14 février à Chaillot ».

L'Humanité, 12 février 1955, « En transit à Paris l'année dernière le célèbre violoniste soviétique Léonide Kogan jouera lundi soir à Chaillot », Georges Léon.

L'Humanité, 14 février 1955, « Un grand violoniste Léonide Kogan jouera ce soir à Chaillot $\gg$.

L'Humanité, 15 février 1955, «Hier soir au Palais de Chaillot un très grand violoniste Léonide Kogan s'est révélé aux Parisiens », Georges Léon.

L'Humanité, 17 février 1955, « Léonide Kogan à Toulouse ».

L'Humanité, 19 février 1955, «Accord parfait de la critique et du public: Léonide Kogan est un très grand violoniste ».

L'Humanité, 26 février 1955, « Léonide Kogan prépare son deuxième récital ».

L'Humanité, 28 février 1955, « Léonide Kogan reçu par la Commission musicale de France-U.R.S.S. ».

L'Humanité, 2 mars 1955, « Léonide Kogan à Nancy ».

L'Humanité, 4 avril 1955, « Deux musiciens français en U.R.S.S. ».

L'Humanité, 30 mai 1955, «Les lauréats du Concours international de violon "Reine Élisabeth" ».

L'Humanité, 31 mai 1955, «Au Palais de Chaillot le 14 juin l'illustre pianiste soviétique Émile Guillels donnera un unique concert ».

L'Humanité, 15 juin 1955, «Ce matin s'ouvre salle Gaveau le 6econcours international Marguerite Long - Jacques Thibaud », Georges Léon.

L'Humanité, 16 juin 1955, « Le concours Marguerite Long - Jacques Thibaud ».

L'Humanité, 17 juin 1955, «Émile Guillels triomphe à Chaillot », Georges Léon, « Le concours Marguerite Long - Jacques Thibaud », « La joie de vivre de Marguerite Long », Georges Léon.

L'Humanité, 18 juin 1955, «Le grand Prix Jacques Thibaud sera décerné aujourd'hui », Georges Léon. 
L'Humanité, 20 juin 1955, «Devy Erlih Grand Prix Jacques - Thibaud », Georges Léon.

L'Humanité, 22 juin 1955, « 27 pianistes au $2^{\mathrm{e}}$ tour du concours Marguerite Long », Georges Léon.

L'Humanité, 24 juin 1955, « Le concours Marguerite Long », Georges Léon.

L'Humanité, 27 juin 1955, « Pas de grand prix Marguerite Long. Bachkirov et Ringeissen seconds grands lauréats », Georges Léon.

L'Humanité, 29 juin 1955, «Distribution des prix Marguerite Long Jacques Thibaud », Georges Léon.

L'Humanité, 30 juin 1955, «Récital franco-soviétique salle Gaveau ».

L'Humanité, 4 juillet 1955, « Trois concerts, six lauréats », Georges Léon.

L'Humanité, 21 juillet 1955, « Un récital dela violoniste polonaise Wanda Wikomirska ». L'Humanité, 30 juillet 1955, «Un éventail de bonne humeur. Les ballets de l'Étoile », Georges Léon.

L'Humanité, $1^{\text {er }}$ août 1955, « Yehudi Menuhin à Moscou... David Oïstrakh à New-York ».

L'Humanité, 22 août 1955, « Le récital Samson François », Georges Léon.

L'Humanité, 13 septembre 1955, «À l'Étoile et aux Champs-Élysées. Ballets », Gilbert Bloch.

L'Humanité, 11 février 1956, « La rencontre de deux grands violoncellistes ».

L'Humanité, 13 février 1956, «Avant son récital de vendredi prochain salle

Gaveau, le grand violoncelliste soviétique Rostropovitch était samedi au Conservatoire », Georges Léon.

L'Humanité, 15 février 1956, « Rostropovitch et ses pairs », Georges Léon.

L'Humanité, 20 février 1956, «Rostropovitch a tenu une gageure à la salle Gaveau », Georges Léon.

L'Humanité, 27 mars 1956, «David Ö̈strakh s'est confirmé le plus grand violoniste vivant », Georges Léon.

L'Humanité, 28 mai 1956, «Les Ballets de Moscou à Paris du 11 juin au 11 juillet », « Préparatifs pour Paris au théâtre Stanislavski de Moscou. Une interview exclusive du Maître de Ballet Vladimir Bourmeister ».

L'Humanité, 29 mai 1956, « Le violoniste soviétique Léonide Kogan vient à Paris révéler un concerto inédit de Vivaldi ».

L'Humanité, 4 juin 1956, « Le violoniste soviétique Léonide Kogan ou le triomphe de la technique et du goût », Georges Léon.

L'Humanité, 9 juin 1956, « Hier au foyer du Châtelet. Première rencontre entre Paris et les danseurs soviétiques ».

L'Humanité, 11 juin 1956, « Pleins feux sur les Étoiles soviétiques ».

L'Humanité, 12 juin 1956, « Première étincelante hier soir au Châtelet ». 
SLOVO

284 Les Voyages lointains des écrivains polonais $\left(\mathrm{XX}^{\mathrm{e}}-\mathrm{XXI}^{\mathrm{e}}\right.$ siècles $)-\mathrm{n}^{\circ} 51$

L'Humanité, 13 juin 1956, «Le "Lac des cygnes” par le ballet Stanislavski », Gilbert Bloch.

L'Humanité, 23 juin 1956, « Les danseurs soviétiques déjeunent avec les critiques parisiens $\gg$.

L'Humanité, 25 juin 1956, «Découverte du Ballet Soviétique avec la troupe Stanislavski », Gilbert Bloch.

L'Humanité, 29 juin 1956, «Les ballets soviétiques au Châtelet "Les Rives du bonheur" », Gilbert Bloch.

L'Humanité, 2 juillet 1956, « "Festival de ballets soviétiques”, au Studio 43 ».

L'Humanité, 5 juillet 1956, «Les Étoiles Soviétiques ont fait danser celles du Marquis de Cuevas », Georges Léon.

L'Humanité, 20 août 1956, « Les Ballets du Grand Théâtre de Moscou viendront l'an prochain à Paris... », Georges Léon.

L'Humanité, 24 août 1956, « Pablo Casals au Festival de Prades », Georges Léon.

L'Humanité, $1^{\text {er }}$ septembre 1956, «Grande danseuse de la musique française moderne Nathalie Trouhanova est morte à Moscou ».

L'Humanité, $1^{\text {er }}$ octobre 1956, «Fièvre à Covent Garden. Les Ballets du GrandThéâtre de Moscou arrivent ».

L'Humanité, 3 octobre 1956, « "Première" soviétique ce soir à Londres ».

L'Humanité, 4 octobre 1956, «Première à Londres hier soir des ballets soviétiques $\gg$, Samuel Lachize.

L'Humanité, 5 octobre 1956, « Enthousiasme dans la presse britannique après la première représentation du ballet Bolchoï », Samuel Lachize.

L'Humanité, 12 octobre 1956, « Pablo Casals : quatre-vingts ans. Cent violoncelles pour un anniversaire », Georges Léon.

\section{L'Humanité-dimanche}

L'Humanité-dimanche, 7 novembre 1948.

L'Humanité-dimanche, 13 novembre 1949, « Antoine et Antoinette dansent aux ballets des Champs-Élysées », Guy Leclerc.

L'Humanité-dimanche, 28 octobre 1951, « Katherine Dunham fait sa rentrée au Théâtre des Champs-Élysées ».

L'Humanité-dimanche, 11 novembre 1951, «Le Grand Ballet du Marquis de Cuevas ».

L'Humanité-dimanche, 25 novembre 1951, «Pour satisfaire le public parisien Katherine Dunham a remanié son spectacle en un temps record », Gilbert Bloch.

L'Humanité-dimanche, 16 décembre 1951, « "La danse au service du peuple" mot d'ordre de Paul et Mathilde Dougnac et de toute leur activité ». 
L'Humanité-dimanche, 14 décembre 1952, « Le grand concert ».

L'Humanité-dimanche, 14 juin 1953, « Trois prestigieux ambassadeurs de l'art soviétique à Paris », Gilbert Bloch.

L'Humanité-dimanche, 21 juin 1953, «Une heure avec la violoniste soviétique Nelly Chkolnikova. Premier grand prix $\mathrm{du}$ concours international Jacques Thibaud », Georges Léon.

L'Humanité-dimanche, 19 juillet 1953, «Musiciens en voyage... Maîtres et disciples soviétiques à travers la France », « De Bruay-en Artois à marseille avec des virtuoses polonais $\gg$, Georges Léon.

L'Humanité-dimanche, 6 septembre 1953, « La mort de Jacques Thibaud ».

L'Humanité-dimanche, 6 décembre 1953, « Le violoniste Igor Ö̈strakh et la presse britannique », « Le violoniste Oïstrakh. La danseuse uzbek Gayla Izmailova ». L'Humanité-dimanche, 13 décembre 1953, «Galina Ismaïlova apporte à Paris les danses d'un Ouzbékistan qu'elle n'a pas connu », D.D.

L'Humanité-dimanche, 27 décembre 1953, « Quand Serge Obratzov directeur du Théâtre des Marionnettes de Moscou parle d'art à ses collègues français », Samuel Lachize.

L'Humanité-dimanche, 14 mars 1954, « Ivan Petrov à l'Opéra ».

L'Humanité-dimanche, 21 mars 1954, «Claude Roy a écouté pour vous Émile Guillels »

L'Humanité-dimanche, 4 avril 1954, «En mai à l'Opéra ».

L'Humanité-dimanche, 11 avril 1954, « Les Maîtres du ballet russe passe dans une salle parisienne ».

L'Humanité-dimanche, 9 mai 1954, «La dernière répétition des Ballets Soviétiques », Claude Roy.

L'Humanité-dimanche, 21 novembre 1954, «Jeune femme des Épinettes Michèle Boussinot passera un an au Conservatoire de Moscou ».

L'Humanité-dimanche, 15 mai 1955, «La semaine culturelle. La création chorégraphique avec : Janine Monin, Igor Fosca, Youra Loboff, Gayle Spear gala présenté par les J.M.F. », J. Laisney.

L'Humanité-dimanche, 27 mai 1956, «Il y a quarante-sept ans tout juste Paris tombait amoureux des danseuses russes $\gg, \mathrm{R}$. L.

L'Humanité-dimanche, 10 juin 1956, « Quarante-sept ans après... Les Ballets russes à Paris ».

L'Humanité-dimanche, 17 juin 1956, «Elles sont les élèves (lointaines) de Marius Petipa ».

L'Humanité-dimanche, 8 juillet 1956. 
SLOVO

286 Les Voyages lointains des écrivains polonais $\left(\mathrm{XX}^{\mathrm{e}}-\mathrm{XXI}^{\mathrm{e}}\right.$ siècles $)-\mathrm{n}^{\circ} 51$

\section{La Pravda}

La Pravda, 2 avril 1937.

\section{La Vie Ouvrière}

La Vie Ouvrière, 22 au 28 décembre 1953.

La Vie Ouvrière, 11 au 17 mai 1954, «Enchantement des ballets soviétiques ».

La Vie Ouvrière, 18 au 24 mai 1954, «Les parisiens ne verront pas les ballets soviétiques $\gg$.

La Vie Ouvrière, 26 juin au 2 juillet 1956, « Les ballets soviétiques ont retrouvé Tchaikovsky », Pierre Delmotte.

\section{Les Lettres françaises}

Les Lettres françaises, 30 septembre 1944, « Malaise chez les musiciens ».

Les Lettres françaises, 16 décembre 1944, « Le souvenir de Serge de Diaguilew », Georges Auric.

Les Lettres françaises, 27 octobre 1945, «Les ballets des Champs-Élysées », Roland-Manuel.

Les Lettres françaises, $1^{\text {er }}$ février 1946, « Le Lac des cygnes », Georges Auric.

Les Lettres françaises, 30 octobre 1947, «Rentrée civile d'un dieu de la musique : Jascha Heifetz », Laurence Audrey.

Les Lettres françaises, 13 novembre 1947, « On dansera ce soir à la République et aux Champs-Élysées », J. Silvant.

Les Lettres françaises, 4 décembre 1947, «Les ballets de Monte-Carlo à l'Alhambra ». J. S.

Les Lettres françaises, 21 juillet 1949, « Le gala de la danse », Victoria Achères.

Les Lettres françaises, 20 octobre 1949, «Le ballet de Monte-Carlo », Victoria Achères.

Les Lettres françaises, 3 novembre 1949, « Ginette Neveu », Henriette Roget.

Les Lettres françaises, $1^{\text {er }}$ décembre 1949, «Du "Tricorne" au "Peintre et son modèle”. Un pas à côté plutôt qu'un pas en avant », Victoria Achères.

Les Lettres françaises, 19 janvier 1950, « À la recherche des voies nouvelles de la musique et de la danse », Henri-Jacques Dupuy.

Les Lettres françaises, 11 mai 1950, « Les Théâtres nationaux », Victoria Achères.

Les Lettres françaises, 8 juin 1950, « À Prades Pablo Casals qui se tait tant que parle Franco, est sorti de sa retraite pour faire entendre J.-S. Bach », Hélène Jourdan-Morhange.

Les Lettres françaises, 22 juin 1950, « Les Ballets Lambert », Victoria Achères. 
Les Lettres françaises, 21 décembre 1950, « L. Oborine, W. Jampolski et I. Bezrodni ou la musique soviétique à Paris », Renaud de Jouvenel.

Les Lettres françaises, 28 décembre 1950, «Le C.N.E. reçoit les deux artistes soviétiques : L. Oborine et I. Bezrodni », Hélène Jourdan-Morhange.

Les Lettres françaises, 28 juin 1951, « Un grand prix international de violon sous le signe de la paix », Jo Heifetz.

Les Lettres françaises, 15 novembre 1951, «La Damnée de Walter Gore », Victoria Achères.

Les Lettres françaises, 13 décembre 1951, « Le prisonnier du Caucase », Victoria Achères.

Les Lettres françaises, 18 au 25 décembre 1952, « Concours international de violon Wieniawski », Hélène Jourdan-Morhange.

Les Lettres françaises, 25 décembre 1952 au $1^{\text {er }}$ janvier 1953, «Palmarès Wieniawski », «Le Grand Concert. Deux heures à l'Opéra de Moscou », Josette Daix.

Les Lettres françaises, 5 au 12 février 1953, « Le Grand Concert », René Jouglet.

Les Lettres françaises, 11 au 18 juin 1953, « David Ö̈strakh est à Paris ».

Les Lettres françaises, 18 au 25 juin 1953, «En écoutant David Ö̈strakh », Paul Grangeon ; «Un concours brillant et juste », Hélène Jourdan-Morhange.

Les Lettres françaises, 25 juin au 2 juillet 1953, « Moins brillant et moins juste », Hélène Jourdan-Morhange.

Les Lettres françaises, 2 au 9 juillet 1953, «Le miracle David Ö̈strakh », Hélène Jourdan-Morhange ; « Rencontre des musiciens », Jean Gandrey-Réty.

Les Lettres françaises, 9 au 16 juillet 1953, « Regrets et rétrospectives », Hélène Jourdan-Morhange.

Les Lettres françaises, 10 au 17 septembre 1953, « Notre Jacques Thibaud », Hélène Jourdan-Morhange ; « Le Monde musical en deuil ».

Les Lettres françaises, 16 au 23 octobre 1953, « Le concert des Maîtres de l'Art russe », Louis Durey.

Les Lettres françaises, 19 au 26 novembre 1953, «Traditionalisme contre tradition », Victoria Achères.

Les Lettres françaises, 10 au 17 décembre 1953, « Tel père, tel fils... Igor Ö̈strakh », Hélène Jourdan-Morhange ; « Brève rencontre... », Paul Grangeon.

Les Lettres françaises, 17 au 24 décembre 1953, «Voici pourquoi Igor Oïstrakh joue si bien... », Hélène Jourdan-Morhange; «Pourquoi Doloukhanova chante si bien... », Irène Joachim; «Voici pourquoi Obrastzov vous fait rire... », Hélène Abraham; «Russes, cosaques et ouzbeks au Théâtre de Paris ou le vertige de la danse », Victoria Achères ; «Comment on devient tambourinaire », Yves Benot. 
SLOVO

288 Les Voyages lointains des écrivains polonais $\left(\mathrm{XX}^{\mathrm{e}}-\mathrm{XXI}^{\mathrm{e}}\right.$ siècles $)-\mathrm{n}^{0} 51$

Les Lettres françaises, 24 au 31 décembre 1953, «La Sonate à Kreutzer de Beethoven », Paul Grangeon.

Les Lettres françaises, 14 au 21 janvier 1954, «Encore une révélation! Annie Fischer », Hélène Jourdan-Morhange, «Diaghilev était aussi critique d'art », Catherine Valogne.

Les Lettres françaises, 18 au 25 février 1954, «Émile Guillels à Paris », Hélène Jourdan-Morhange.

Les Lettres françaises, 4 au 11 mars 1954, «Émile Guillels ou l'égalité dans la perfection », Hélène Jourdan-Morhange ; « Guillels au C.N.E. ».

Les Lettres françaises, 11 au 18 mars 1954, « Ivan Petrov: Un Boris sans pareil », Paul Grangeon.

Les Lettres françaises, 18 au 25 mars 1954, «Ivan Petrov. Un grand Méphisto qui conduit le bal », Jean Gandrey-Réty; «Vlado Perlemuter », Hélène Jourdan-Morhange.

Les Lettres françaises, $1^{\text {er }}$ au 8 avril 1954, «Hommage à J. Thibaud par les jeunes lauréats soviétiques », Hélène Jourdan-Morhange.

Les Lettres françaises, 8 au 15 avril 1954, «La sereine tranquillité de Nelly Chkolnikova », Hélène Jourdan-Morhange.

Les Lettres françaises, 15 au 22 avril 1954, «Les étoiles du ballet russe », Victoria Achères.

Les Lettres françaises, 29 avril au6 mai 1954, « Galina Oulanova, en route pour Paris. "Comment j'ai appris mon métier...” », Galina Oulanova.

Les Lettres françaises, 6 au 13 mai 1954, «La danse décomposée par Galina Oulanova », Victoria Achères.

Les Lettres françaises, 30 septembre au 7 octobre 1954, « Le Sadler's Wells Ballet à l'Opéra », Victoria Achères.

Les Lettres françaises, 4 au 11 novembre 1954, «Étoiles des ballets russes », Victoria Achères.

Les Lettres françaises, 17 au 24 février 1955, «Leonid Kogan à Chaillot », Hélène Jourdan-Morhange.

Les Lettres françaises, 10 au 17 mars 1955, «Léonid Kogan ou la maîtrise de l'archet », Hélène Jourdan-Morhange.

Les Lettres françaises, 17 au 24 mars 1955, «Premiers jours à Varsovie », Hélène Jourdan-Morhange.

Les Lettres françaises, 24 au 31 mars 1955, « Le Concours Chopin à Varsovie », Hélène Jourdan-Morhange.

Les Lettres françaises, 23 au 30 juin 1955, «Le concours Jacques ThibaudMarguerite Long », Hélène Jourdan-Morhange. 
Les Lettres françaises, 30 juin au 7 juillet 1955, «Au Concours Marguerite Long - Jacques Thibaud ».

Les Lettresfrançaises, 17 au 23 novembre 1955, « Une performanced'Arthur Rubinstein ». Les Lettres françaises, 24 au 30 novembre 1955, « Retour d'Ivan Pétrov », Jean Gandrey-Réty.

Les Lettres françaises, 23 au 29 février 1956, « Rostropovitch », Hélène Jourdan-Morhange.

Les Lettres françaises, 29 mars au 4 avril 1956, «Ö̈strakh à Paris », Hélène Jourdan-Morhange.

Les Lettres françaises, 7 au 13 juin 1956, «Un grandissime violoniste », Hélène Jourdan-Morhange.

Les Lettres françaises, 14 au 20 juin 1956, «Léonid Kogan aux ChampsÉlysées », « Ballets russes au Châtelet. De la "Première" de mai 1909 à celle de juin $1956 »$, Maurice Lehmann.

Les Lettres françaises, 21 au 27 juin 1956, «Les ballets soviétiques », Victoria Achères.

Les Lettres françaises, 28 juin au 4 juillet 1956, « Deux ballets qui ne se ressemblent pas », Victoria Achères.

Les Lettres françaises, 5 au 11 juillet 1956, « Ballets soviétiques », Victoria Achères.

Les Lettres françaises, 18 au 24 octobre 1956, «Les 80 ans de Pablo Casals », Hélène Jourdan-Morhange.

\section{Literatournä̈a Gazeta}

Literatournaïa Gazeta, 24 avril 1952, « Le Ballet et l'Actualité », Igor Moïsseïev.

\section{Regards}

Regards, 15 février 1945, « Roland Petit va danser “Guernica” », Yves Bonnat.

Regards, $1^{\mathrm{er}}$ mars 1945, « The Sadlers Wells Ballet vient danser à Paris ».

Regards, 11 juillet 1947, « Héritière de la Pavlova la danseuse de la Mort du Cygne

Ludmilla Tcherina a cherché un sosie, elle a trouvé une marionnette ».

Regards, 13 octobre 1949, « Aux ballets de Monte-Carlo. On parle des langues

différentes mais on danse d'un même cœur », Yves Bonnat.

Regards, 18 novembre 1949, « Le ballet d'Elsa Triolet », Yves Bonnat.

Regards, 1956, « Le Lac des Cygnes retrouve Paris ».

\section{Sovietskö̈ Isskoustvo}

Sovietskoë Isskoustvo, 12 juin 1951. 
SLOVO

290 Les Voyages lointains des écrivains polonais $\left(\mathrm{XX}^{\mathrm{e}}-\mathrm{XXI}^{\mathrm{e}}\right.$ siècles $)-\mathrm{n}^{\circ} 51$

\section{Autres références}

Jouvenel Renaud (de), mars 1951, « Le "Chant des forêts" de Chostakovitch et la musique soviétique » in La Nouvelle Critique, n² 24, p. 92-99.

Lemaire Frans C., 2004, La Musique du XX siècle en Russie et dans les anciennes Républiques soviétiques, Fayard, Paris, 521 p.

LÉON Georges, mai 1954, « "Le Concert des Maîtres de l'art” » in La Nouvelle Critique, ${ }^{\circ} 55$, p. 255-261.

Soria Georges, août-septembre 1954, «Les échanges culturels avec le monde socialiste $\gg$ in Cabiers du communisme, $\mathrm{n}^{\circ}$ 8-9, p. 1028-1040. 